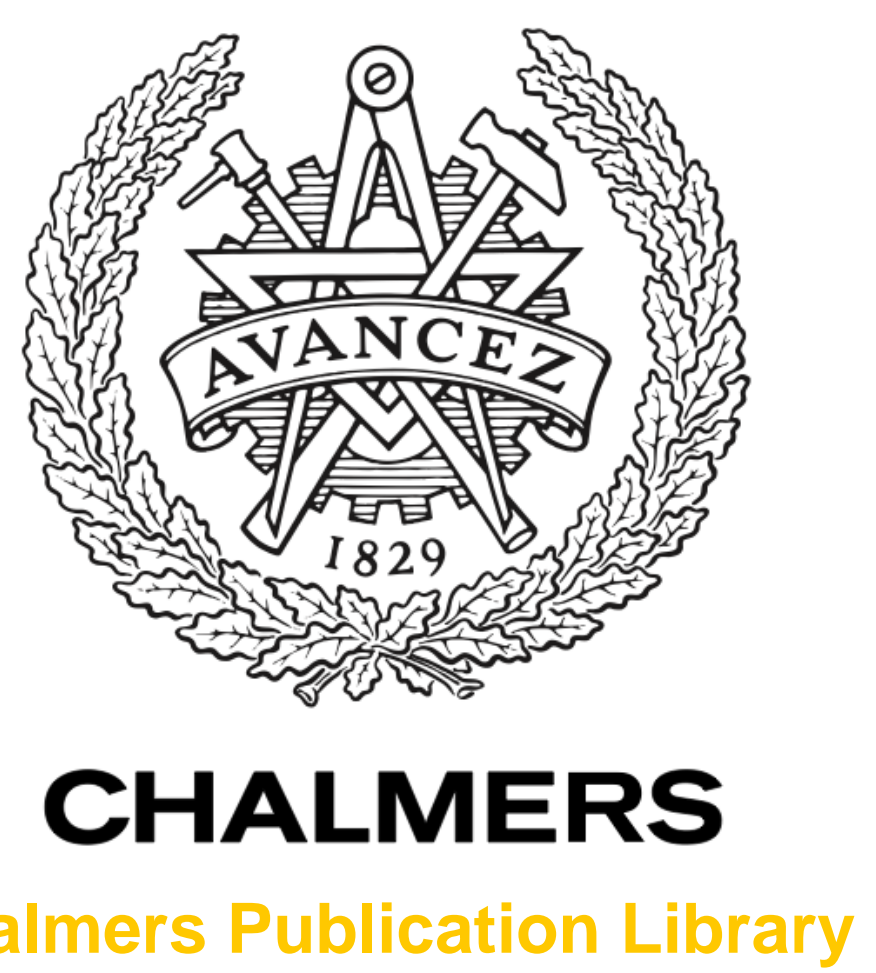

\title{
Quantitative numerical analysis of flow past a circular cylinder at Reynolds number between 50 and 200
}

This document has been downloaded from Chalmers Publication Library (CPL). It is the author's version of a work that was accepted for publication in:

Journal of Fluids and Structures (ISSN: 0889-9746)

Citation for the published paper:

Qu, L. ; Norberg, C. ; Davidson, L. (2013) "Quantitative numerical analysis of flow past a circular cylinder at Reynolds number between 50 and 200". Journal of Fluids and Structures, vol. 39 pp. $347-370$.

http://dx.doi.org/10.1016/j.jfluidstructs.2013.02.007

Downloaded from: http://publications.lib.chalmers.se/publication/180053

Notice: Changes introduced as a result of publishing processes such as copy-editing and formatting may not be reflected in this document. For a definitive version of this work, please refer to the published source. Please note that access to the published version might require a subscription. 


\title{
Quantitative numerical analysis of flow past a circular cylinder at Reynolds number between 50 and 200
}

\author{
Lixia $\mathrm{Qu}^{\mathrm{a}}$, Christoffer Norberg ${ }^{\mathrm{b}, *}$, Lars Davidson ${ }^{\mathrm{c}}$, Shia-Hui Peng ${ }^{\mathrm{c}, \mathrm{d}}$, Fujun Wang ${ }^{\mathrm{a}, * *}$ \\ ${ }^{a}$ China Agricultural University, 100083 Beijing, China \\ ${ }^{b}$ Department of Energy Sciences, Lund Institute of Technology, SE-221 00 Lund, Sweden \\ ${ }^{c}$ Chalmers University of Technology, SE-412 96 Gothenburg, Sweden \\ ${ }^{d}$ Swedish Defence Research Agency (FOI), SE-164 90 Stockholm, Sweden
}

\begin{abstract}
Results of numerical simulations are presented for flow past a stationary circular cylinder at low Reynolds numbers ( $\mathrm{Re}=50-200)$. The simulations were carried out using a finitevolume code employing a fractional step method with second-order accuracy in both space and time. A sensitivity study on numerical parameters concerning the domain size, grid independence and time step resolution was carried out in detail for $\mathrm{Re}=100$. Global timeaveraged results on force coefficients, non-dimensional velocities and pressures, including their corresponding r.m.s. values, as well as various quantities related to the separation and vortex shedding characteristics are presented. A non-monotonous streamwise velocity recovery in the intermediate wake is observed for $\operatorname{Re}>50$, a phenomenon that has been grossly overlooked in the past. There are two plateaus along the wake centerline, in particular for $\operatorname{Re}=200$. The first, which is the most distinct, ranges from about $x=9$ to $x=16$ at a wake deficit velocity of $0.38, x$ being counted in diameters behind the cylinder axis; the second one appears from $x=25$ to $x=28$ at a wake deficit velocity of 0.54 . This phenomenon seems to be related to an associated change-over in the orientation of the von Kármán vortices and the merging trends, especially for $\mathrm{Re}=200$ beyond $x=25$, as observed from instantaneous vorticity fields. Three-dimensional simulations using spanwise lengths of 10 and 12 (diameters) were carried out at $R e=200$. After a long initial phase with regular three-dimensional mode A flow features increasing very slowly in amplitude, the flow went into a state with distinct pulsating forces acting on the cylinder, the pulsations
\end{abstract}


being seemingly randomly localized across the cylinder span. In this second, much more chaotic, flow state, the time-averaged results were in agreement with previous experiments and with parts of previous numerical studies.

Keywords: Circular cylinder, Incompressible flow, Numerical simulation, Low Reynolds number, Vortex shedding

\section{Introduction}

The flow past a circular cylinder has been intensively investigated, mainly because of its fundamental importance and its relevance for practical applications. Due to the circular shape of the cylinder, which means that the location of flow separation is only influenced by the flow regime or the upstream conditions, the accuracy is a challenge for numerical study.

Depending on the Reynolds number, the flow behavior in the cylinder wake can be generally classified as laminar, transitional or turbulent. Williamson (1996b) defined various shedding regimes according to the variation of the base suction coefficient over a large range of Reynolds numbers. In the so-called laminar shedding regime, which for sufficiently long cylinders ranges from about $R e \simeq 47.4$ (Norberg, 1994; Kumar and Mittal, 2006) to Re $\simeq 165-190$, the latter limit being hysteretic and somewhat dependent on the end conditions (Norberg, 1994; Williamson, 1995), there is a large scatter in the results reported in the literature, especially about the drag coefficient. We are trying to figure out the reason.

In this study, all physical lengths are scaled with the cylinder diameter, velocities with the oncoming free stream velocity, physical times with the diameter divided by the free stream velocity, and pressure differences with the dynamic pressure of the oncoming flow, which is assumed to be steady and uniform.

Most previous numerical studies in the laminar shedding regime have concentrated on the phenomenon of vortex shedding in the near-wake region $(0<x<10$, approximately). Except for some very few studies, e.g. Inoue and Yamazaki (1999), it seems that the subsequent wake recovery towards the free stream condition has been largely overlooked. As

${ }^{*}$ Tel: (+46)46-2228606. E-mail address: christoffer.norberg@energy.lth.se.

**Tel: (+86)10-62736972; Fax: (+86)10-62736972. E-mail address: wangfj@cau.edu.cn. 
details of this recovery might be of importance for the subsequent instabilities that occur in the very far wake, e.g. see Cimbala (1984) and Williamson and Prasad (1993), we will present detailed distributions of the time-averaged streamwise velocity along the wake centerline $(y=0, x>0)$, in particular for $\operatorname{Re}=150$. Moreover, in the vast majority of simulation studies, the emphasis has been on introducing new computational techniques and code validation. The results reported are then often restricted to one or two Reynolds numbers, for 2-D shedding flow mostly $\mathrm{Re}=100$ and/or $\mathrm{Re}=200$. Notable exceptions from such studies are Henderson (1995), Park et al. (1998), Baranyi and Lewis (2006), Stålberg et al. (2006), and Posdziech and Grundmann (2007). As is well known, local and global results may be influenced by various numerical parameters or factors. A high accuracy of the results demands for not only a sufficiently fine spatial resolution but also a sufficiently fine time resolution. Further, the required necessary minimum domain distances will be dependent on the Reynolds number, as well as on the applied boundary conditions.

The selection of physical domain is a common problem in numerical simulation of cylinder flow. The domain size and computational cost must be balanced to obtain the best results. For 2-D simulations with a strictly polar or O-grid domain around the cylinder, the crosssectional domain size can be defined by a single parameter, e.g. the outer diameter, $D$. A rectangular domain is more flexible and also more natural with respect to the specification of boundary conditions. It can generally be described by three parameters: the distance $X_{u}$ from the cylinder center to the inlet or upstream boundary, the corresponding distance $X_{d}$ to the outlet or downstream boundary, and the lateral distance $H$ between the upper and lower boundaries, see Figure 1. If at lateral boundaries the transverse (normal) velocity component is set to zero, the flow case can be regarded as being influenced by a blockage or confinement effect. For rectangular domains, the blockage parameter then is $H^{-1}$ (confinement parameter $H$ ). In 3-D simulations, the domain is simply extended in the spanwise direction, as specified with its linear spanwise dimension, here denoted $L_{z}$. For a rectangular domain, Behr et al. (1995) investigated the influence of the location of the lateral boundaries on 2-D computation of unsteady incompressible flow past a circular cylinder at $\operatorname{Re}=100$. They found, by employing a traction-free condition at the outflow boundary and slip-wall consitions at the 
lateral boundaries, that the lateral boundaries should be removed from the cylinder by at least a distance of eight cylinder diameters, i.e. that at least $H>16$ is required. Kumar and Mittal (2006) pointed out that the onset of vortex shedding is significantly influenced by the confinement parameter $H$. A rectangular domain was used in their study, while a slip-wall boundary condition was employed at lateral boundaries $(H=8-200)$. When using a domain with $X_{u}=X_{d}=50$, the effects of blockage (or confinement) were found negligible only for cases with $H>100$. Shi et al. (2004) demonstrated for Re $=100$ that a polar domain greater than $D=100$ was needed to get results that were more or less independent of this parameter. On the outlet boundary condition when using such large domains, they found no essential difference when using the non-reflective boundary condition and the pure Neumann (zero-gradient) boundary condition. In addition, when the downstream distance is sufficiently long, it seems that the convective outlet condition performs in a similar manner as the traction-free condition (Park et al., 1998). Since the independence from the domain extensions seems to be an arbitrary assumption on the part of of many authors, Posdziech and Grundmann (2007) used extremely large domain sizes to search for asymptotic solutions for domain independence. They applied Neumann conditions at the outlet using a constant value of $X_{d}=50$ for various extensions of inflow length and height, from 20 up to 4000 . They concluded that, for any case, $X_{u}$ should be larger than 20. They showed that, in contrast to the Strouhal number, the force coefficient and the base suction coefficient are strongly dependent on the resolution and even more on the size of the computational domain. In particular when using $H=140$ and $H=8000$ and for laminar shedding conditions, Posdziech and Grundmann (2007) presented high-order polynominal functions on the Re-dependencies for the drag coefficient $C_{D}$, the base suction coeffiient $-C_{p b}$, the Strouhal number St and the amplitude coefficient of the sinusoidal lift variations $\hat{C}_{L}=\sqrt{2} C_{L^{\prime}}\left(C_{L^{\prime}}\right.$ is the r.m.s. lift coefficient).

The accuracy of the numerical results depends strongly on the resolution of the velocity shear layers near the cylinder walls. The adapted numerical method with different spatial order of convergence has different requirements with regard to the location of the first grid point. For instance, Franke et al. (1990) reported that the first grid point away from the wall 
(radial distance specified as $\delta$ ) has a particularly strong influence on the results, especially on the Strouhal number. For a circular cylinder using a polar domain with $D=40$ and a Neumann condition at the outlet, $\delta \simeq 0.001$ were found to be sufficient ( $\operatorname{Re}=50-5000)$. Mittal (2005) used the finite element mesh for his computations, with a thickness of the first layer of 0.001 , for Re from 50 to 350. Li et al. (2009) obtained a grid independent solution by setting $\delta=1 / 160$ for $\operatorname{Re}=50-300$, using a volumetric Lattice-Boltzmann boundary approach. The required cell size close to the cylinder surface is also dependent on the Reynolds number. A lower Reynolds number case requires more uniform grid distributions in the overall sense, whereas a very fine mesh density is required close to the walls at higher Reynolds number, due to viscous effects. The studies mentioned above probably simply used the same grid at different Reynolds numbers for convenience.

Obviously, the choice of time step, which is known to depend on the order of convergence of the numerical time marching scheme, also plays an important role in numerical simulation of vortex shedding flows. Implicit schemes are usually preferred because there are no stability restrictions on the time step, the only restriction then being governed by the desired accuracy. Baranyi and Lewis (2006) introduced two methods to simulate low Reynolds number cylinder flow, namely a grid-based CFD method and a vortex dynamics method, using time steps of 0.0005 and 0.05, respectively, to get similar results. Posdziech and Grundmann (2007) used a computational time step of $\Delta t=0.005$ in all computations to study the domain size effect $(\operatorname{Re}=50-250)$. Mittal $(2001)$ carried out computations for a cylinder at $\operatorname{Re}=100$, by setting the time step as 0.25. In his later paper (Mittal, 2005) for $R e=50-100$, a time step size of 0.0625 was utilized for all the computations, while adapting the same numerical procedure. The above studies adapted totaly different time steps, mainly because of different time marching schemes.

Since the reliability and accuracy of numerical results are strongly dependent on the above numerical parameters, our first objective is to investigate these from various 2-D simulations at a typical Reynolds number in the laminar shedding regime, $R e=100$. The reason for choosing this particular Re is that it is by far the one most investigated, which means that there is a solid basis for comparisons. Our second objective refers to the lack of reliable data 
for various quantities in the laminar shedding regime. In particular, we will present results on time-averaged quantities for $\mathrm{Re}=50-200$ based on variations around the cylinder surface (pressure and vorticity) and along the wake centerline (pressure and streamwise velocity). The final objective is to investigate the effects of flow three-dimensionality at $R e=200$. From experiments at this Re, e.g. see Williamson (1988); Norberg (1994); Williamson (1995), the natural state is in the early transition regime, where the flow is highly three-dimensional. Williamson (1992) refers to this state as mode $A^{*}$.

\section{Simulation Methodology}

As incompressible viscous flow with constant fluid properties was assumed, the sole governing parameter, except for geometrical domain restrictions, is the Reynolds number, Re, traditionally based on the cylinder diameter and the uniform oncoming free stream velocity.

\subsection{Domain and Mesh}

As shown in Figure 1, the circular cylinder was placed in a rectangular computational domain with its center at the origin of a Cartesian coordinate system; coordinates $x, y, z$ indicate streamwise, cross-stream and spanwise directions, respectively. Boundary conditions (Section 2.2) are also shown in the figure. Since the present computational domain was square, the domain size can be defined by $H$, the height of the domain. Hence, the distances of the upstream and downstream boundaries and side walls from the center of the cylinder can all be specified as $H / 2\left(X_{u}=X_{d}=H / 2\right)$. In addition, the spanwise distance was specified as $L_{z}$.

Structured meshes created by an in-house code called G3DMESH were used, see Eriksson (1985). The grid topology is depicted in Figure 2. For Reynolds numbers beyond 80, a polar grid of radius 20 (units) was adapted in a sub-domain enclosing the cylinder, see Figure 2(b). For the lower Reynolds number cases $(\mathrm{Re}=50,60,80)$, in order to get more orthogonal grid distributions, a radius of 40 was used for the polar sub-domain. The polar grid was stretched in the radial direction with a Cubic Hermite grid point distribution. Outside the polar grid 


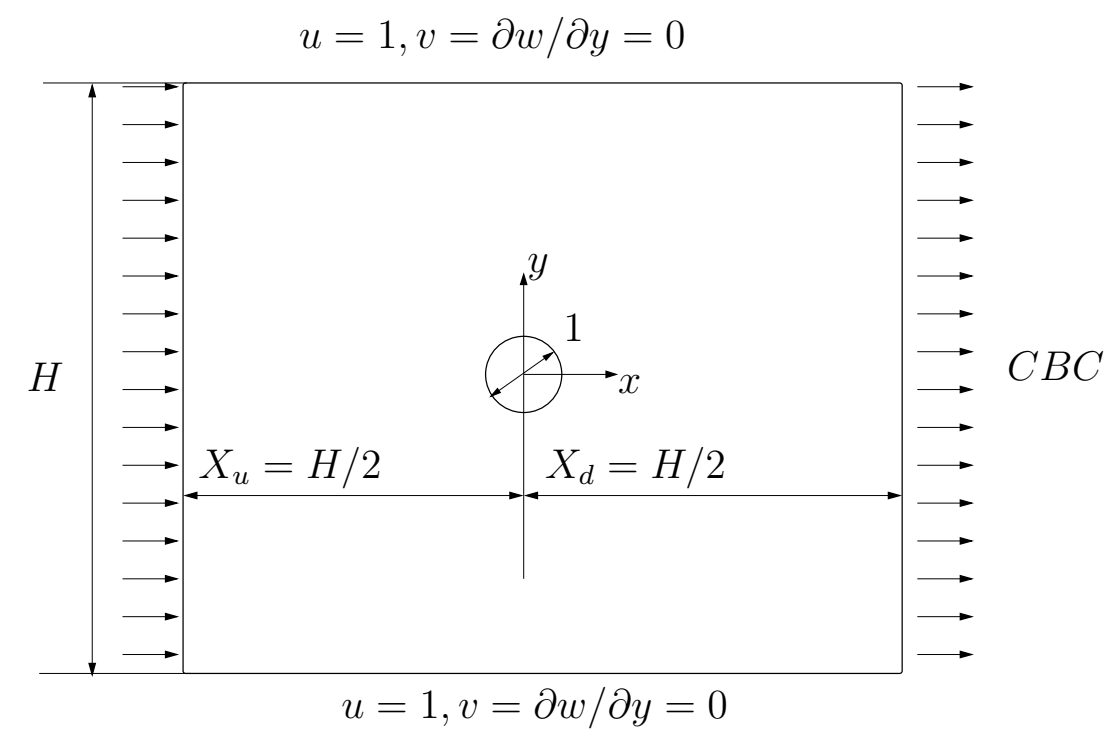

Figure 1: Problem definition for flow past a stationary circular cylinder.

region, the grid was gradually merged into a quadratic region in four radial sectors, see Figure 2(a). For the study of domain independence (Section 4.1.1), the inner (polar) mesh distribution was left unaltered. Considering the importance of the wake region, but also to some extent in the decelerating parts upstream of the cylinder, a biased accumulation of grid points was applied in these regions, see Figure 2(a).

The mesh can be defined by three parameters, $N_{i}, N_{j}, N_{k}$, which indicate the number of grid points in the circumferential, radial and axial directions, respectively. For 2-D cases, the mesh can thus be specified only with two global parameters, $N_{i}$ and $N_{j}$. For the case referred to as $3-\mathrm{D}(\operatorname{Re}=200$, see Table 4$)$, spanwise distances of $L_{z}=10$ and $L_{z}=12$ were used, with 128 and 160 uniformly distributed cells $\left(N_{k}=162\right.$ and $\left.N_{k}=130\right)$, respectively. For cell sizes in the transverse $x y$-plane, they can be roughly specified by two parameters, the distance of the first grid away from the cylinder surface, $\delta$, and the streamwise cell size at the outlet section close to $y=0, \delta_{e}$.

\subsection{Numerical details}

An incompressible, finite volume code was used (Davidson and Farhanieh, 1995; Davidson and Peng, 2003). The numerical procedure is based on an implicit, fractional step technique with a multigrid pressure Poisson solver (Emvin, 1997) and a non-staggered grid 


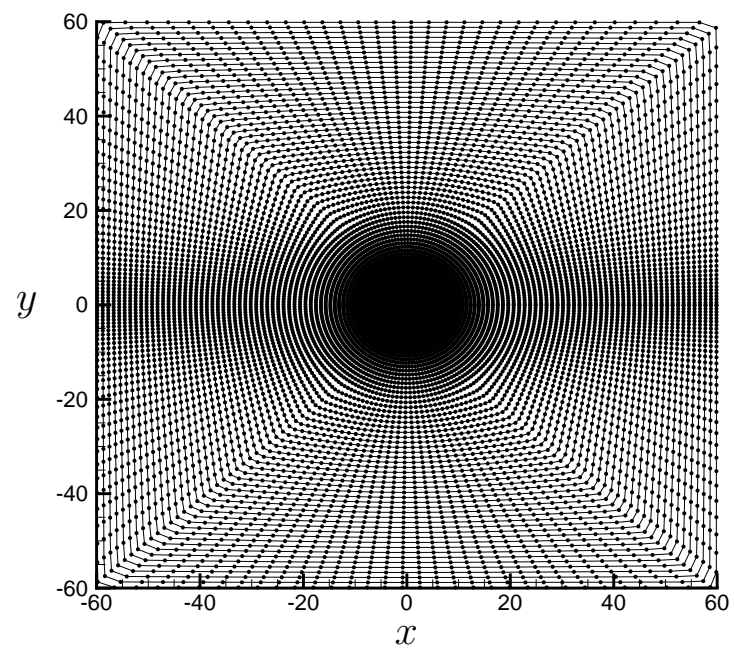

(a)

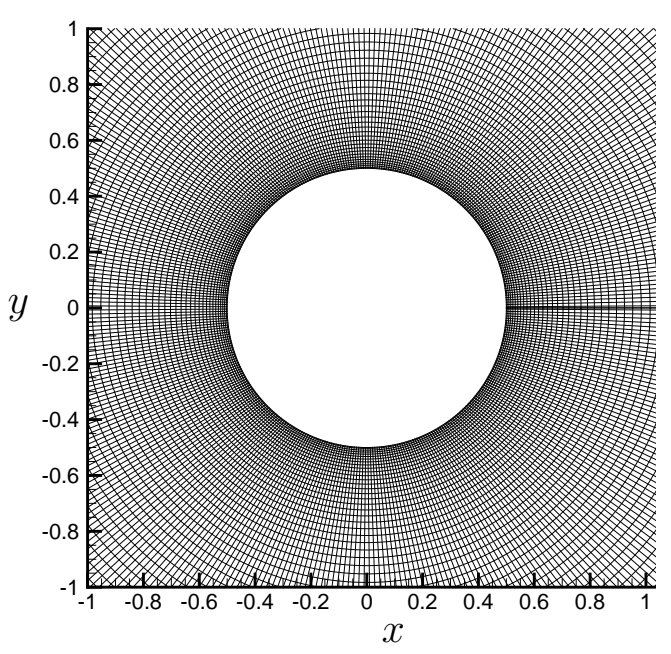

(b)

Figure 2: Sketch of numerical grid, $H=120, N_{i}=386, N_{j}=322, \delta=0.0054, \delta_{e}=0.26$. (a) full domain; (b) near-cylinder close-up (polar grid).

arrangement. Second-order central differencing is used in space, and the second-order CrankNicolson scheme is used in time.

The inflow was treated as a uniform free stream, $u=1, v=w=0$. No-slip conditions were applied on the cylinder surface, $u=v=w=0$. Symmetry boundary conditions were imposed in the spanwise direction, $\partial u / \partial z=\partial v / \partial z=w=0$. Lateral boundary conditions were set as $u=1, v=\partial w / \partial y=0$, called the free stream condition (Posdziech and Grundmann, 2007). A convective boundary condition was applied at the outlet, described as

$$
\frac{\partial u_{i}}{\partial t}+C \frac{\partial u_{i}}{\partial x}=0
$$

where $i$ indicates velocity components $u(i=1), v(i=2)$ and $w(i=3)$. As recommended for vortex shedding flow (Yoshida et al., 1993; Sohankar et al., 1998), the synthetic outlet convective speed was set equal to the free stream velocity, $C=1$.

The convergence criterion was set to $10^{-4}$. Tests with smaller values of this precision, e.g. $10^{-5}$, showed no changes in the results. Convergence was assessed at the end of each 
iteration on the basis of the residual sources criterion, which compares the sum of the absolute residual source with some reference values, typically taken as the inlet flux of the relevant variable fed into the domain of calculation (Davidson and Farhanieh, 1995; Sohankar et al., 1999). An efficient multigrid method was used to solve the Poisson equation for pressure correction. Hence, only a few iterations (typically two) solving the momentum equations and the Poisson pressure equations were required at each time step to obtain convergence. More details can be found in Davidson and Peng (2003). The minimum number of iterations for each time step was set to three although in general only two iterations were enough to satisfy the convergence criterion.

The time integration interval for calculating time-averaged quantities was chosen within the saturated state in which, for the 2-D cases, the time history of lift and drag coefficients behaved almost perfectly sinusoidally. For all 2-D cases in this study the integration time included at least 80 (time-averaged) vortex shedding cycles. The criterion (a posteriori) for determining the beginning of the saturated state was based on the time variation of the drag coefficient, which was found to be more conservative than using the lift signal. It can be noted that the time required to reach the saturated state was strongly dependent on the Reynolds number; it took almost 700 time units for $\mathrm{Re}=50$, whereas it only took about 80 and 60 time units for $\mathrm{Re}=100$ and $\mathrm{Re}=200$ (2-D cases), respectively. Interestingly, for the 3 -D case $\left(\operatorname{Re}=200, L_{z}=12\right)$, and after an initial start-up period of about 60 time units, the flow remained quite a long time in a state that appeared to be saturated with highly ordered but rather weak mode A (Williamson, 1996a; Thompson et al., 2001) instability structures. However, after about 500 time units, the flow gradually (within a time interval of about 100 units) went in to another state with more disordered but still mode-A related flow structures. Time-averaged quantities for this particular case were calculated from this last flow state and were integrated from $t=680$ to $t=1240$ corresponding to about 100 time-averaged shedding cycles. For the case with $L_{z}=10$, the time spent in the ordered but still 3-D state was even longer. Similar experiences with rather long start-up times from 3-D simulations in this regime can be found in Posdziech and Grundmann (2001) $(\operatorname{Re}=210)$ and Braza et al. (2001) $(\mathrm{Re}=220)$. As clarified in Posdziech and Grundmann (2001), the 
main sources of errors with regard to the numerical results are found in the spanwise lengths, domain extensions, resolution and integration time. Especially for the transition regime, it is rather difficult to obtain accurate and trustworthy results.

\section{Validation}

\subsection{Definition of global quantities}

The bulk or global quantities were all calculated using commonly accepted definitions. Time-averaged velocities are denoted with an overbar, e.g. $\bar{u}$ for the streamwise velocity. r.m.s. values (standard deviations of fluctuations) are denoted with a prime, e.g. $u^{\prime}$ for the r.m.s. streamwise velocity. The reference pressure for the pressure coefficient, $C_{p}$, was taken from the inlet section. The base suction coefficient, $-C_{p b}$, refers to the negative of the time-averaged $C_{p}$ at the base of the cylinder $(x=0.5, y=0)$. For convenience and to avoid redundant digits, the time-averaged $C_{p}$ at the front of the cylinder $(x=-0.5, y=0), C_{p s}$, is reported as $a_{s}=\left(C_{p s}-1\right)$ Re, the scaling being based on that $a_{s}=4$ for a stagnation line in plane stagnation flow towards a circular cylinder at high enough Re, see Homann (1936a). Time-mean drag $\left(C_{D}\right)$ and lift $\left(C_{L}\right)$ coefficients include pressure $\left(C_{D p}, C_{L p}\right)$ and frictional $\left(C_{D f}, C_{L f}\right)$ components. The fluctuations of lift, drag and pressure coefficients are presented using r.m.s. values $\left(C_{L^{\prime}}, C_{D^{\prime}}, C_{p^{\prime}}\right)$. For convenience, the ratio between the coefficients of r.m.s. lift and r.m.s. drag is denoted $r$, i.e. $C_{D^{\prime}}=C_{L^{\prime}} / r$. The Strouhal number, St, the inverse value of the non-dimensional time-mean shedding period, was detected by FFT of the time history of the lift coefficient. Since the force coefficients behave sinusoidally for 2-D cases, the corresponding frequency was also calculated directly from the time series of lift coefficients by detecting peak values and/or zero-crossing values. Both procedures gave the same results, within $\pm 0.1 \%$.

The wake closure length, $L_{c}$, was determined as the second point of vanishing $\bar{u}$ along the wake centerline, from negative to positive values. Angular positions of time-mean separation, $\theta_{s}$, were calculated from points of zero wall vorticity $\left(\omega_{z}=\partial v / \partial x-\partial u / \partial y=0\right)$ along the cylinder surface. This definition is exact for steady 2-D flow, whereas for unsteady flow it should, according to Sears and Telionis (1975), be defined as the point of simultaneous 
vanishing of the shear stress and velocity at a point within the wall-bounded shear layer. Braza et al. (1986) checked both methods within a shedding period for $R e=100$ and found that the difference is less than $0.3 \%$ (less than $0.35^{\circ}$ ).

\subsection{Comparison with previous numerical results}

Table 1 presents a comparison of present and previous global numerical results for $\mathrm{Re}=$ 100; the present ones were obtained with $(H=120$, mesh $386 \times 322, \Delta t=0.005)$. The comparison studies in Table 1 all have comparable domain sizes.

Table 1: Comparison of flow quantities with earlier numerical results; $\operatorname{Re}=100$.

\begin{tabular}{lccccccc}
\hline Author(s) (year) & $-C_{p b}$ & $C_{D p}$ & $C_{D}$ & $L_{c}$ & $\mathrm{St}$ & $C_{L^{\prime}}$ & $r$ \\
\hline Park et al. (1998) & 0.725 & 0.99 & 1.33 & 1.42 & 0.165 & 0.235 & 36.5 \\
Kravchenko and Moin (1998) & 0.73 & 0.99 & 1.32 & 1.45 & 0.164 & 0.222 & - \\
Shi et al. (2004) & - & - & 1.318 & - & 0.1640 & - & - \\
Mittal (2005) & - & - & 1.322 & - & 0.1644 & 0.226 & 35.6 \\
Stålberg et al. (2006) & - & 0.972 & 1.32 & - & 0.166 & 0.233 & 36.7 \\
Posdziech and Grundmann (2007) & 0.709 & - & 1.325 & - & 0.1644 & 0.228 & - \\
Li et al. (2009) & 0.701 & 0.995 & 1.336 & - & 0.164 & - & - \\
Present, D9 & 0.709 & 0.984 & 1.319 & 1.41 & 0.1648 & 0.225 & 36.0 \\
\hline
\end{tabular}

Park et al. (1998) used a finite volume method and employed a C-grid system with a periodic boundary condition at the branch cut and a convective outflow boundary condition. A Dirichlet boundary condition was used at the far field boundaries. The domain size was $X_{u}=50, X_{d}=20, D=100$ (polar part). Kravchenko and Moin (1998) used a zonal Bspline method. The case in Table 1, which refers to their case 9, has a O-type zonal mesh of diameter $D=120$. The boundary conditions were similar to the ones used in Park et al. (1998). The results chosen from Shi et al. (2004) were obtained by employing a finite volume method using a convective (non-reflective) condition at the outflow boundary, with domain size $D=300$ in a circular domain. Mittal (2005) carried out finite element simulations with a rectangular domain $\left(H=100, X_{u}=X_{d}=50\right)$. A Neumann-type boundary condition with a zero viscous stress vector was prescribed at the downstream boundary. The flow was 
allowed to slip on the lateral boundaries of the domain. Stålberg et al. (2006) used a high order finite difference method and put the far field boundary at 40 diameters away from the cylinder $(D=80)$, with grid number of $130 \times 80$. The grid used was of O-type mapped by a polar coordinate transformation. Posdziech and Grundmann (2007) used a spectral code on a rectangular domain with $X_{d}=50$; upstream $\left(X_{u}\right)$ and lateral $(H / 2)$ distances were between 20 and 4000. A free stream condition was applied at lateral boundaries; a Neumann condition for velocities and zero pressure were prescribed at the outflow boundary. Please note that their results in Table 1 are for $H=140$. Unless otherwise noted, our subsequent comparison results of Posdziech and Grundmann (2007) are also valid for this domain size $\left(H=140, X_{u}=70, X_{d}=50\right)$. Li et al. (2009) used the lattice-Boltzmann method (LBM) and placed the inlet, outlet and lateral boundaries at 50 units away from the cylinder center $\left(H=100, X_{u}=X_{d}=50\right)$. Free stream conditions with a constant pressure were imposed on the upstream and downstream boundaries and periodicity on lateral boundaries.

When considering the various different boundary conditions, numerical methodologies, domain sizes etc., the present results fit well with the comparison cases presented in Table 1. However, it can be noted that domain sizes in general are much smaller than the ones displayed in Table 1. The importance of having large enough domains has been emphasized by Posdziech and Grundmann (2007). Although not all quantities in Table 1 can be found, the overall conclusion is that the present results compare favorably with those of Posdziech and Grundmann (2007).

\section{Results and discussion}

We start with the sensitivity study at $\mathrm{Re}=100$ in Section 4.1 , with regard to domain size, grid and time step, and then analyze the effect of Reynolds number at $\operatorname{Re}=50-200$ in Section 4.2. The streamwise wake-velocity deficit is described and briefly discussed in Section 4.3, while the 3-D results at $\mathrm{Re}=200$ are presented in Section 4.4. 


\subsection{Sensitivity study at $\operatorname{Re}=100$}

\subsubsection{Effect of domain size}

Results for different domain sizes are compared here, using 386 cells in the circumferential direction, which was proven to be good enough to capture the near cylinder flow features (Section 4.1.2). The time step was $\Delta t=0.02$; see cases D1-D4 in Table 2. The cell size close to the cylinder was $\delta=0.0054$; for cases D1, D2 and D4, $\delta_{e}=0.26$, for case D3, $\delta_{e}=0.25$. For cases with a smaller domain size, such as $H=60$, there was an increase in the drag coefficient by about $1.2 \%$ and $1.9 \%$ for $C_{L^{\prime}}$, compared to $H=200$. This was probably caused by the greater blockage (Kang, 2006; Posdziech and Grundmann, 2007). The base pressure coefficients increased by about $3 \%$ when the domain was decreased from $H=200$ to $H=60$. The pressure coefficient at the front stagnation, the bubble size behind the cylinder and the separation angles were all constant for the different domains, which indicate that those quantities are not sensitive to the domain size $(H>60)$.

From Figure 3(a) it can be observed that the mean streamwise velocity has the same reduction trend along the wake centerline for different domains, while no significant difference can be observed in the near wake region $(0<x<10)$. The r.m.s. pressure coefficients along $y=0$ are shown in Figure 3(b), which have rather consistent trends, except for the case of the shortest domain, $H=60$. When the outlet location was shorter than $X_{d}=60$, some spurious fluctuations occurred towards the outlet section. These fluctuations became rather significant for the case with $X_{d}=30(H=60)$, affecting the wake flow upstream to about $x=15$. As the wake deficit decreased somewhat near the outlet, this ought to decrease the drag; however, the blockage effect was probably greater so that, in total, there was only a slight increase in drag. The increased blockage also caused some slight increases in r.m.s. lift and Strouhal number.

From this subsection we conclude that the domain size of $H=120$ is good enough for $\operatorname{Re}=100$. When compared with $H=200$, the maximum deviation for the Strouhal number was about $0.8 \%$; the deviations for all other quantities in Table 2 were less than $0.5 \%$. 
Table 2: Global results from two-dimensional simulations; $\mathrm{Re}=100$.

\begin{tabular}{cccccccccccc}
\hline Case & $H$ & $N_{i} \times N_{j}$ & $\Delta t$ & $C_{D}$ & $C_{D p}$ & $r$ & $C_{L^{\prime}}$ & $-C_{p b}$ & $L_{c}$ & $\theta_{s}\left[^{\circ}\right]$ & $\mathrm{St}$ \\
\hline D1 & 200 & $386 \times 482$ & 0.02 & 1.310 & 0.976 & 37.1 & 0.2151 & 0.697 & 1.44 & 118.0 & 0.1647 \\
D2 & 120 & $386 \times 322$ & 0.02 & 1.315 & 0.979 & 37.1 & 0.2163 & 0.703 & 1.44 & 118.0 & 0.1650 \\
D3 & 100 & $386 \times 290$ & 0.02 & 1.317 & 0.981 & 37.1 & 0.2169 & 0.706 & 1.44 & 118.0 & 0.1652 \\
D4 & 60 & $386 \times 210$ & 0.02 & 1.326 & 0.988 & 37.0 & 0.2191 & 0.719 & 1.44 & 118.0 & 0.1660 \\
D5 & 120 & $482 \times 322$ & 0.02 & 1.315 & 0.980 & 37.0 & 0.2165 & 0.704 & 1.44 & 117.9 & 0.1651 \\
D6 & 120 & $322 \times 322$ & 0.02 & 1.314 & 0.978 & 37.2 & 0.2160 & 0.702 & 1.45 & 118.0 & 0.1649 \\
D7 & 120 & $242 \times 322$ & 0.02 & 1.312 & 0.977 & 37.6 & 0.2148 & 0.700 & 1.45 & 118.0 & 0.1645 \\
D8 & 120 & $386 \times 418$ & 0.02 & 1.316 & 0.978 & 37.4 & 0.2131 & 0.701 & 1.46 & 117.5 & 0.1647 \\
D9 & 120 & $386 \times 322$ & 0.005 & 1.319 & 0.984 & 36.0 & 0.2253 & 0.709 & 1.41 & 118.0 & 0.1648 \\
D10 & 120 & $386 \times 322$ & 0.01 & 1.317 & 0.982 & 36.2 & 0.2224 & 0.707 & 1.42 & 118.0 & 0.1649 \\
D11 & 120 & $386 \times 322$ & 0.04 & 1.309 & 0.973 & 38.8 & 0.2031 & 0.696 & 1.49 & 117.9 & 0.1652 \\
\hline
\end{tabular}

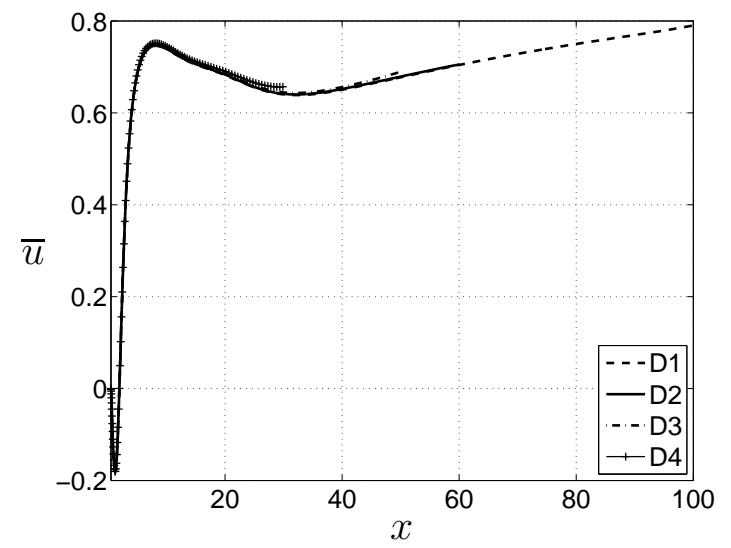

(a)

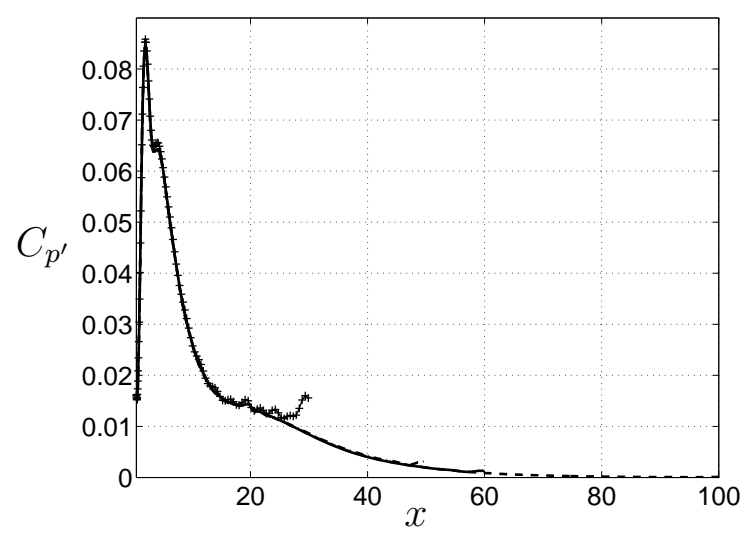

(b)

Figure 3: Distributions of mean streamwise velocity and r.m.s. of pressure coefficient for different domains, $\operatorname{Re}=100$.

\subsubsection{Grid sensitivity study}

The grid sensitivity study was carried out for domain size $H=120$; see cases D2, D5-D8 in Table 2. In cases D2, D5-D7, which have different grid numbers in the circumferential direction $\left(N_{i}\right)$ but the same $N_{j}=322$, all variations were within $\pm 0.6 \%$, except ratio $r=$ $C_{L^{\prime}} / C_{D^{\prime}}$ for which there was an increase of $1.6 \%$ with a decrease from $N_{i}=482$ (case D5) 
to $N_{i}=242$ (case D7); the associated decrease in $C_{D^{\prime}}$ was $2.4 \%$. When also considering variations along the cylinder surface and in the very near wake, the judgement was here that $N_{i}=386$ was required to capture all the essential near-cylinder flow features (corresponding to an angular wall resolution of $0.93^{\circ}$ ). Cases D2 and D8 have the same $N_{i}=386$ but case $\mathrm{D} 8$ is better resolved in the radial direction $\left(N_{j}=418, \delta=0.003, \delta_{e}=0.22\right)$. In this comparison, all quantities in Table 2 were within $\pm 0.5 \%$, except $C_{L^{\prime}}$ and wake bubble length $L_{c}$, for which, when comparing case D8 with case D2, the relative changes were $-1.5 \%$ and $+1.2 \%$, respectively. This implies that these two quantities are somewhat sensitive to the overall grid density, and especially in the radial direction. However, as the associated changes in drag coefficients and in Strouhal number were indeed small, it was judged that grid $386 \times 322$ could be used in the subsequent simulations, albeit with suitable adjustments due to Reynolds number on $\delta$ and domain size $H$, to account for the anticipated changes in the thickness of the wall-bounded shear layers $(\delta)$ and in the overall viscous-affected region around the cylinder $(H)$, respectively.

\subsubsection{Effect of time step}

The study of the influence of the time step was carried out for domain size $H=120$ using mesh $386 \times 322$; see cases D9, D10, D2 and D11 $(\Delta t=0.005$ to $\Delta t=0.04)$ in Table 2 and Figure 4. The corresponding maximum CFL (Courant-Friedrichs-Lewy) numbers were around $0.58,1.1,2.3$ and 4.6 , respectively.

There were only small to negligible influences from the time step on $C_{D p}, C_{D}, \theta_{s}$ and St, with changes within the percentage level. When the time step was increased from $\Delta t=0.005$ (D9) to $\Delta t=0.04$ (D11), the r.m.s. lift decreased by $9.8 \% ; L_{c}$ was prolonged by about $5.6 \%$ and $-C_{p b}$ dropped by $1.8 \%$; the ratio $r=C_{L^{\prime}} / C_{D^{\prime}}$ increased by $7.8 \%$, which means that $C_{D^{\prime}}$ decreased by as much as about $16 \%$. When the time step was set to $\Delta t=0.1,0.2$, the results deviated far from the experimental and other simulation results, and thus those results were not considered credible. When comparing with the most time-resolved case (case D9, $\Delta t=0.005$ ), it can be noted that all variations when doubling this time step (case $\mathrm{D} 10, \Delta t=0.01)$ were within the percentage level. 
Figure 4(a) shows that all time steps produced similar trends along $y=0$. The mean streamwise velocity fluctuations along $y=0$ show no significant difference beyond $x>4$, while, in the near wake region Figure 4(b), it is clear that the time-averaged flow is somewhat affected by the time step. Figure 4(c) shows that the surface pressure fluctuations change significantly with the time step used; for mean surface pressures (not shown), the associated variations were hardly discernible. It can be noted that the upper and lower (shoulder) sides of the cylinder are more sensitive to the time step than the front and rear. Since these shoulder parts (with the nearby flow separation) are strongly associated with the fluctuating lift (Norberg, 2003), it is not surprising that the r.m.s. lift coefficient is quite sensitive to the time step. Figure 4(d) illustrates that the pressure fluctuation level in the whole near wake decreases with an increase in time step, a reflection that the separated shear layers are periodically swept through this region. In addition, Figure 4 indicates that the r.m.s. pressure coefficients converge at a somewhat slower rate than the time-averaged velocity. To some part, a reason for this is pure statistics. Second-order quantities need longer integration times to converge compared to time-mean (first-order) quantities.

For the present simulations, the number of iterations per time step was about the same for all cases tested, which means that the total computer time for each case was approximately proportional to $\Delta t$. To achieve reasonable computer times in the next step when studying the influence of Reynolds number, and still be in the position to produce reasonably accurate results, a time step of $\Delta t=0.01$ was selected for further simulations (except where noted otherwise). 


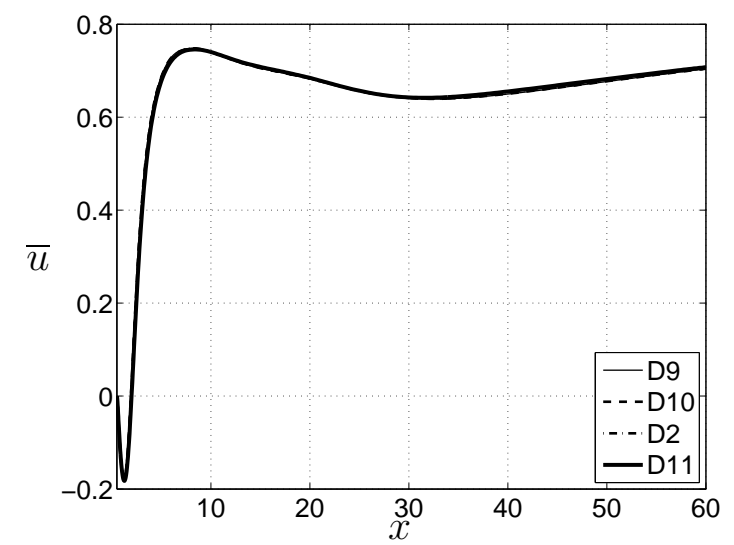

(a)

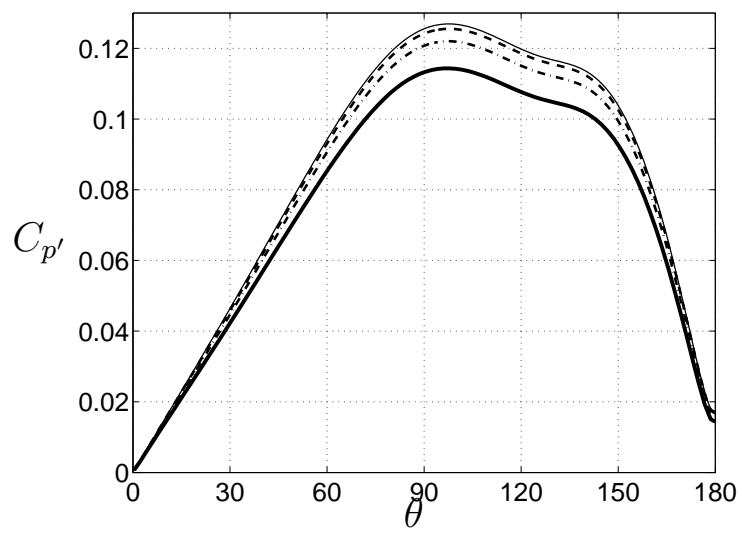

(c)

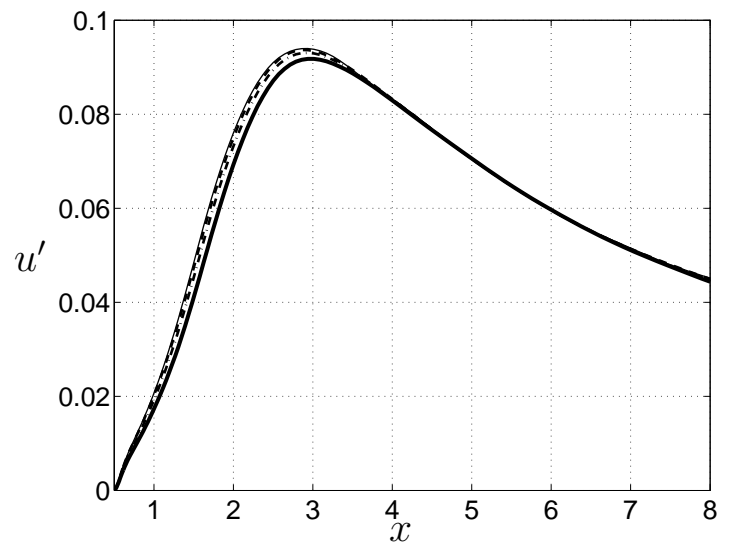

(b)

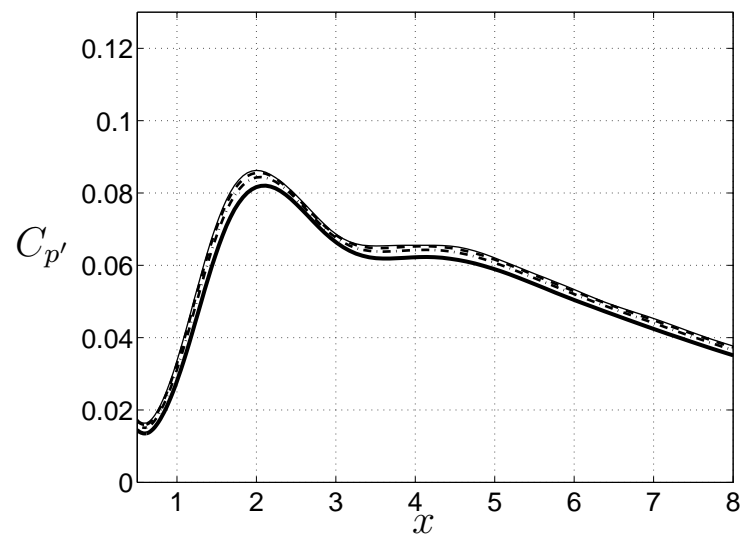

(d)

Figure 4: Comparison of velocity and r.m.s. pressure distributions for different time steps, $\operatorname{Re}=100$ : (a) streamwise mean velocity; (b) r.m.s. of streamwise velocity. (c) r.m.s. of the pressure coefficients on the cylinder surface; (d) r.m.s. of pressure coefficients along the wake centerline.

\subsection{Effect of Reynolds number; two-dimensional simulations}

This section gives results from 2-D simulations for different Reynolds numbers within the range $50-200$. The domain size was partly adjusted according to the scale of $1 / \sqrt{R e}$, while keeping the same number of nodes. At $\operatorname{Re}=50$, we have the cell sizes $\delta=0.01, \delta_{e}=0.49$, while, at $\operatorname{Re}=60,80$, we have the cell sizes $\delta=0.008, \delta_{e}=0.37$. For $\operatorname{Re}=100,120$, we use the same domain, where $\delta=0.0054, \delta_{e}=0.26$. Then, for $\operatorname{Re}=150-200$, the cell sizes are $\delta=0.0048, \delta_{e}=0.24$. 
Table 3: Global results for different Reynolds numbers, mesh $386 \times 322, \Delta t=0.01$.

\begin{tabular}{ccccccccccc}
\hline $\mathrm{Re}$ & $H$ & $a_{s}$ & $C_{D}$ & $C_{D p}$ & $r$ & $C_{L^{\prime}}$ & $-C_{p b}$ & $L_{c}$ & $\left.\theta_{s}{ }^{\circ}\right]$ & $\mathrm{St}$ \\
\hline 50 & 200 & 5.11 & 1.397 & 0.943 & 327 & 0.0399 & 0.511 & 2.46 & 124.0 & 0.1240 \\
60 & 160 & 5.43 & 1.377 & 0.956 & 134 & 0.0871 & 0.560 & 2.11 & 123.0 & 0.1354 \\
80 & 160 & 5.53 & 1.336 & 0.967 & 56.9 & 0.1616 & 0.640 & 1.67 & 120.3 & 0.1528 \\
100 & 120 & 5.73 & 1.317 & 0.982 & 36.2 & 0.2224 & 0.707 & 1.42 & 118.0 & 0.1649 \\
120 & 120 & 5.73 & 1.306 & 0.997 & 27.1 & 0.2776 & 0.764 & 1.25 & 116.4 & 0.1739 \\
150 & 100 & 5.70 & 1.305 & 1.024 & 20.5 & 0.3546 & 0.846 & 1.06 & 114.7 & 0.1841 \\
150 & 160 & 5.70 & 1.301 & 1.021 & 20.6 & 0.3529 & 0.840 & 1.06 & 114.7 & 0.1837 \\
180 & 100 & 5.62 & 1.310 & 1.051 & 17.0 & 0.4251 & 0.921 & 0.93 & 113.5 & 0.1919 \\
200 & 100 & 5.51 & 1.316 & 1.068 & 15.5 & 0.4678 & 0.966 & 0.86 & 112.9 & 0.1958 \\
\hline
\end{tabular}

\subsubsection{Global time-averaged results}

Table 3 shows the detailed global results for different Reynolds numbers. The timeaveraged pressure coefficient at the frontal stagnation point (line) of the cylinder, in Table 3 read-out as $C_{p s}=1+a_{s} /$ Re, decreased with increasing Re. The coefficient $a_{s}$ reached a local maximum value within $\mathrm{Re}=100-120$. To the authors' knowledge and for laminar shedding conditions, the frontal pressure coefficient $C_{p s}$ has not been explicitly presented in earlier numerical investigations. However, for $\mathrm{Re}=100$, there are some investigations in which the time-averaged $C_{p}$ around the cylinder is presented, the values being $a_{s} \approx 5$ (Park et al., 1998; Sharman et al., 2005). For laminar shedding flow, the experimental values from Thom $(1928,1933)$ and Homann $(1936 \mathrm{~b})$ are within $a_{s}=2.3-8.4$.

The drag coefficients decrease with increasing Reynolds number to $R e=120$, then follows a minor increase from $\mathrm{Re}=150$ to $\mathrm{Re}=200$, see also Figure 5. Other indicators vary with Reynolds number monotonously. In Norberg (1994), it is mentioned that at a Reynolds number lower than 250, where the vortex formation is laminar, an increase in St is associated with an increase in the base suction coefficient $\left(-C_{p b}\right)$. The results in Table 3 show agreement with this.

Moreover, the increase in Re is associated with a reduction in the wake bubble length $L_{c}$ 
and an upstream movement of the separation line, in agreement with e.g. Park et al. (1998) and $\mathrm{Wu}$ et al. (2004), see also Figure 8. As evident from Table 3, the wake closure point moves upstream with increasing Re in conjunction with an increase in base suction, and that the vortex shedding formation occurs closer to the cylinder base at higher Reynolds number, see Figure 13. Within $\mathrm{Re}=50-200$, the wake bubble length, $L_{c}$, decreases by about $65 \%$. Obviously, at higher Reynolds numbers, the convection effect on the flow gets stronger while the viscous effect gets weaker. Balachandar et al. (1997) analyzed the force balance on the mean separation bubble in the wake of a circular cylinder, with a wide range of Reynolds numbers from 250 to 140000. They concluded that the Reynolds normal stress outweighs the net force from the shear stress, so that the net pressure force tends to push the bubble away from the body. However, in the present low Reynolds number cases, viscosity plays quite an important role, which means that viscous forces cannot be ignored as in Balachandar et al. (1997), especially for the lower Reynolds numbers. Hence, the stretching effect of viscous shear stresses can partly explain why $L_{c}$ becomes smaller at higher Reynolds number. In addition, the increase with Re of the time-averaged normal stresses due to shedding-related velocity fluctuations, the laminar equivalent of Reynolds normal stresses, probably outweighs the pressure force in this laminar shedding regime where the corresponding non-viscous shear stresses are quite small, contributing to pushing the bubble towards the body. Consequently, the von Kármán vortices are formed closer to the base of the cylinder with increasing Reynolds number.

As reported in Norberg (1998), from Re $\simeq 1000$ up to $R e \simeq 6000$, the product of vortex formation length and base suction coefficient $\left(-C_{p b}\right)$ was approximately equal to 1.8 , whereas, for greater Reynolds numbers, the value instead was about 1.6. However, in the present study in the laminar vortex shedding regime, the product of the streamwise wake closure distance $\left(x_{c}=L_{c}+0.5\right)$ and corresponding base suction $\left(-C_{p b}\right)$ varied from 1.51 to 1.31, with respect to Reynolds numbers from 50 up to 200. Even though this product value was not really a constant as in the turbulent shedding regime mentioned above, the variation was quite small, which is believed to be related to viscous effects.

Comparing the total drag coefficient, $C_{D}$, and the pressure drag coefficient, $C_{D p}$, it can 


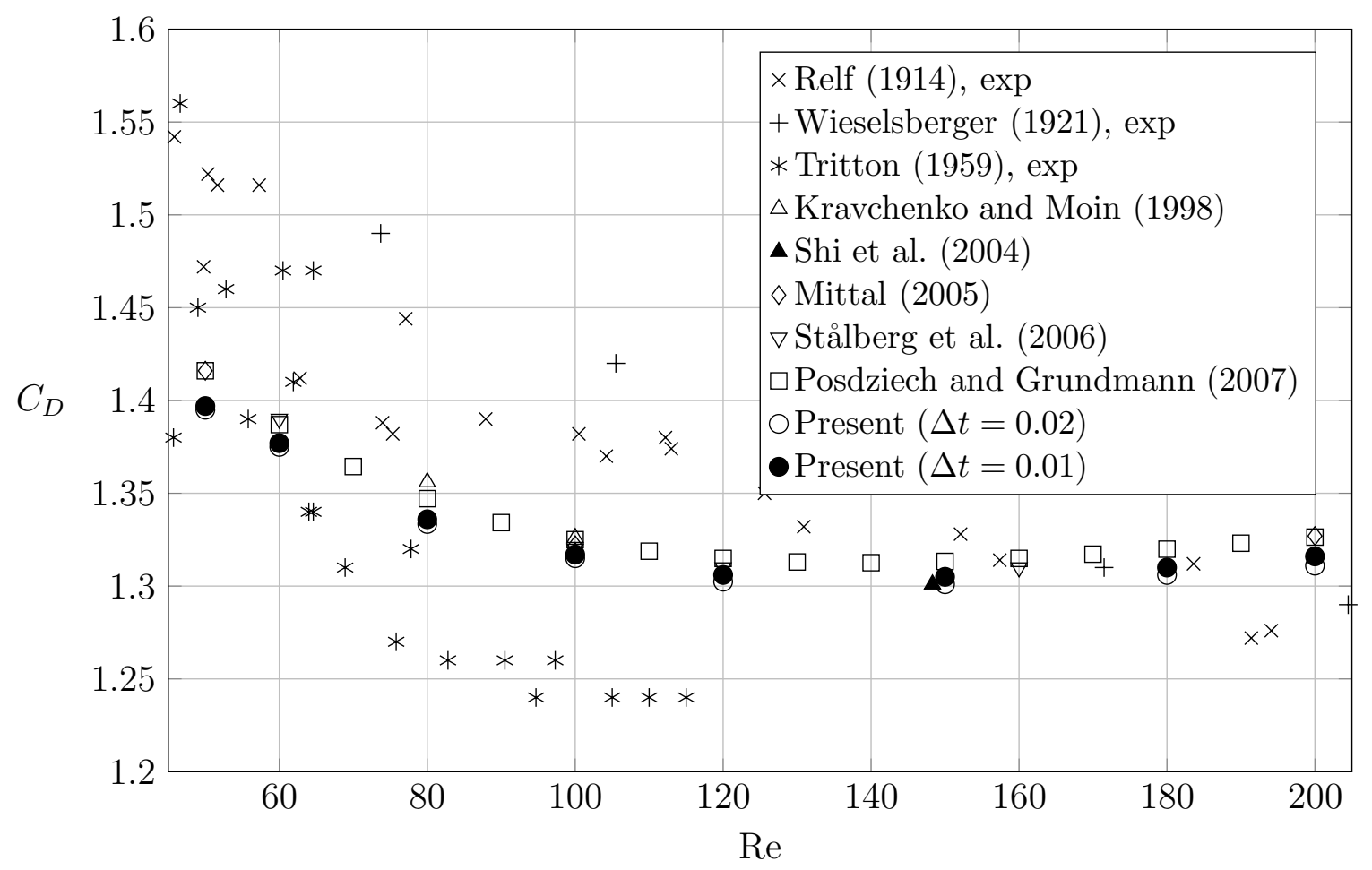

Figure 5: Time-averaged drag coefficient versus Reynolds number.

be seen that $C_{D p}$ increases with Reynolds number, getting more dominant. Meanwhile, the friction component of the drag coefficient, $C_{D f}=C_{D}-C_{D p}$, drops with Re roughly as $C / \sqrt{\operatorname{Re}}$, where $C=3.21$ at $\operatorname{Re}=50$, and $C=3.51$ at $\operatorname{Re}=200$. A large scatter of gathered drag coefficients can be seen in Figure 5, both experimental and numerical results. Since laboratory experiments for low Re often have strict requirements, such as spanwise aspect ratios, end conditions and the accuracy of instrumentation, it might be unavoidable to get scattered data. On the other hand, possible reasons for the deviation of numerical results could be small computational domains, spatial/temporal resolution problems, and the boundary conditions applied. In addition, the numerical results that are gathered from code validation studies can partly cause the scatter due to different numerical methods. It should be noted that present results are nearly parallel to the constant-blockage data of Posdziech and Grundmann $(2007)(H=140)$. If instead compared to their drag coefficients obtained with $H=4000$, i.e. negligible blockage, almost perfect agreement can be observed 
(not shown).

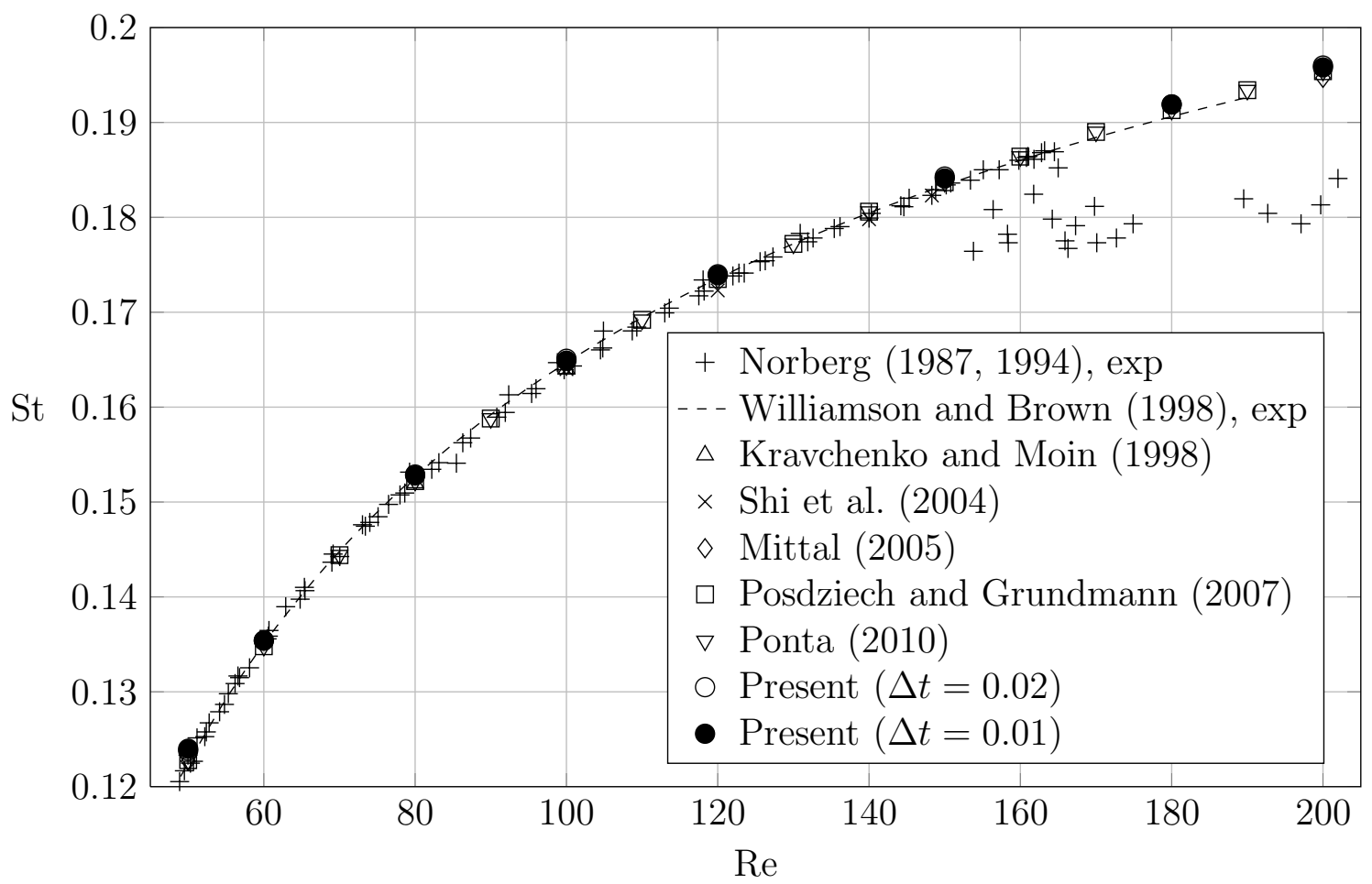

Figure 6: Strouhal number versus Reynolds number.

As is well known, the Strouhal number increases rapidly with Re in the laminar shedding regime (Roshko, 1954), see Figure 6. It can be seen that the present Strouhal numbers fit very well with previous results. Please note that, in the experiments of Norberg (1987, 1994), it was not possible to maintain laminar vortex shedding flow for $\operatorname{Re}>165$. As discussed e.g. in Williamson (1995), this is most likely due to the influence of the end plates used in these experiments. By slightly manipulating the end conditions, it is possible to maintain continuous laminar vortex shedding flow up to about $\operatorname{Re}=190$, e.g. see Williamson (1995). The correlation inserted as another experimental reference is taken from the study of Williamson and Brown (1998), based on the experiments that can be found in Williamson $(1988,1989)$.

The r.m.s. lift coefficient, $C_{L^{\prime}}$, increases rapidly with increasing Re (Figure 7 ) due to stronger alternate periodic vortex shedding. At the lower Re end, the ratio $r=C_{L^{\prime}} / C_{D^{\prime}}$ 
decreases dramatically with increasing Re, which manifests a completely different initial Re dependency for the variation of $C_{D^{\prime}}$ when compared to $C_{L^{\prime}}$. For $C_{L^{\prime}}$ at $\operatorname{Re}=50,60$, the inferred initial behavior was proportional to $\sqrt{\mathrm{Re}}$. In fact, for both $\operatorname{Re}=50$ and $\operatorname{Re}=60$, the $C_{L^{\prime}}$ could be represented as $0.17 \sqrt{\epsilon}$, where $\epsilon=\left(\operatorname{Re}-\operatorname{Re}_{c}\right) / \operatorname{Re}_{c}$ using $\operatorname{Re}_{c}=47.4$ (Norberg, 1994; Kumar and Mittal, 2006). At higher Re, in particular for Re $\geq 100$, the r.m.s. lift variation could be very well approximated with the formula as presented in Norberg (2001), $C_{L^{\prime}}=\sqrt{\epsilon / 30+\epsilon^{2} / 90}$, again using $\operatorname{Re}_{c}=47.4$. As seen in Figure 7 , the fluctuating lift due to alternating wall pressures already dominates at the lower Re end; the ratio $C_{L^{\prime} p} / C_{L^{\prime}}$ increases from 0.84 at $\operatorname{Re}=50$ to 0.92 at $\operatorname{Re}=200$. Over the whole interval, the ratio between the coefficients of r.m.s. lift due to wall friction and total r.m.s. lift, $C_{L^{\prime} f} / C_{L^{\prime}}$, showed a decrease that was proportional to the inverse square root of the Reynolds number, the proportionality factor being $c_{1}=1.30( \pm 1 \%)$. As inferred from Park et al. (1998), there is a phase shift between the lift fluctuations due to pressure and friction. For sinusoidal lift signals and with a constant phase shift of $\phi$ between the frictional and pressure parts, the relation between the r.m.s. lift coefficients can be written as

$$
C_{L^{\prime}}^{2}=C_{L^{\prime} f}^{2}+C_{L^{\prime} p}^{2}+2 C_{L^{\prime} f} C_{L^{\prime} p} \cos \phi
$$

From inspection of the signals within $\operatorname{Re}=50-200$, it was found that the phase $\phi$ was almost constant, $\phi \simeq 30^{\circ}$, which means that wall-frictional lift leads its pressure counterpart by about $1 / 12$ of a shedding period. The (positive and constant) phase difference is probably related to the maximum wall friction occurring upstream of the shoulder area, an area in which the lift due to pressure has its main origin. The maximum wall pressure fluctuation is located at around $\theta=(84 \pm 3)^{\circ}$, whereas the maximum time-mean wall vorticity is at around $(51 \pm 1)^{\circ}$ (as can be seen in Figure 11). In Figure 7, the results of Posdziech and Grundmann (2007) for $C_{L^{\prime}}$ using their largest domain $(H=4000)$, which compare favorably with the present ones, have been used to calculate (i) the inferred $C_{L^{\prime} f}$ using $c_{1}=1.3$, and (ii) the inferred $C_{L^{\prime} p}$ using a constant phase of $\phi=30^{\circ}$, in agreement with the present results in these quantities. 


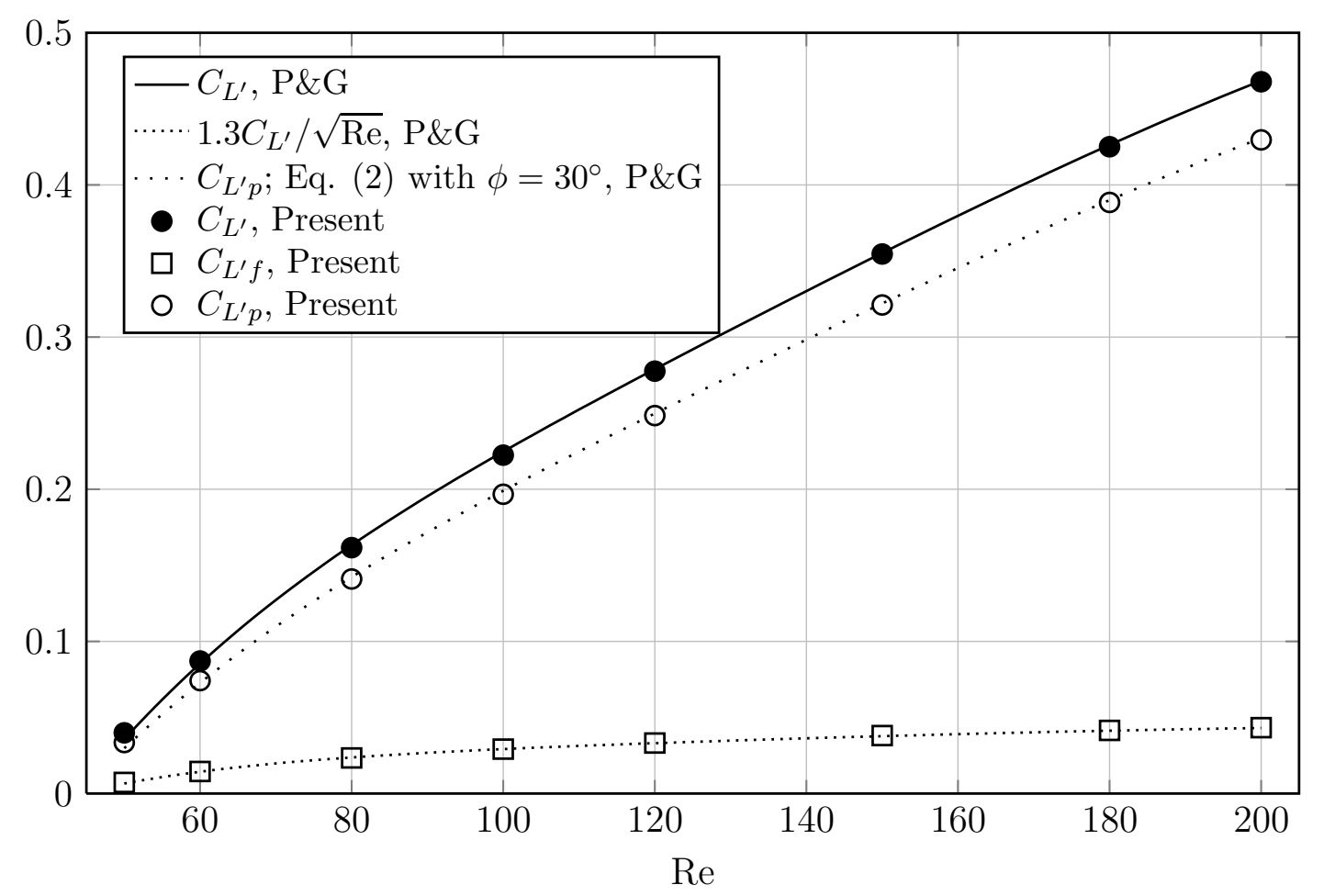

Figure 7: R.m.s. lift coefficients vs. Re. P\&G = Posdziech and Grundmann (2007), $H=4000$.

The time-mean of separation angles, $\theta_{s}$, are displayed in Figure 8. The separation points move upstream with increasing Reynolds number, associated with the decreasing thickness of the wall-bounded separated shear layers, due to stronger vortex shedding (visible in Figure 13). The instantaneous separation angle fluctuation amplitudes (small symbols) around the time-averaged separation angle are shown in Figure 8. The location of the separation angle oscillates periodically in time as shown by Braza et al. (1986) and Baranyi and Shirakashi (1999). The present simulation results are shown as open circles including error bars (small open circles), which demonstrate the maximum oscillation range of the instantaneous separation angle around the individual mean value for a given Reynolds number. Due to the oscillating character of the vortex-shedding process, plus the convective effect tending to outweigh the viscous effect, the variation in the instantaneous separation angle with time is greater for a higher Reynolds number case. Comparing with the numerical results of Park et al. (1998) and experimental results of Wu et al. (2004), similar trends can be observed. For 
instance, the time-averaged separation angle decreases as the Reynolds number increases for the presently studied Reynolds number range; the variations of the instantaneous separation angle with time are greater for a higher Reynolds number. The empirical equation for the separation angle and Reynolds number relationship $(10 \leqslant R e \leqslant 200)$ suggested by Wu et al. (2004) is also given in this figure as the dashed line.

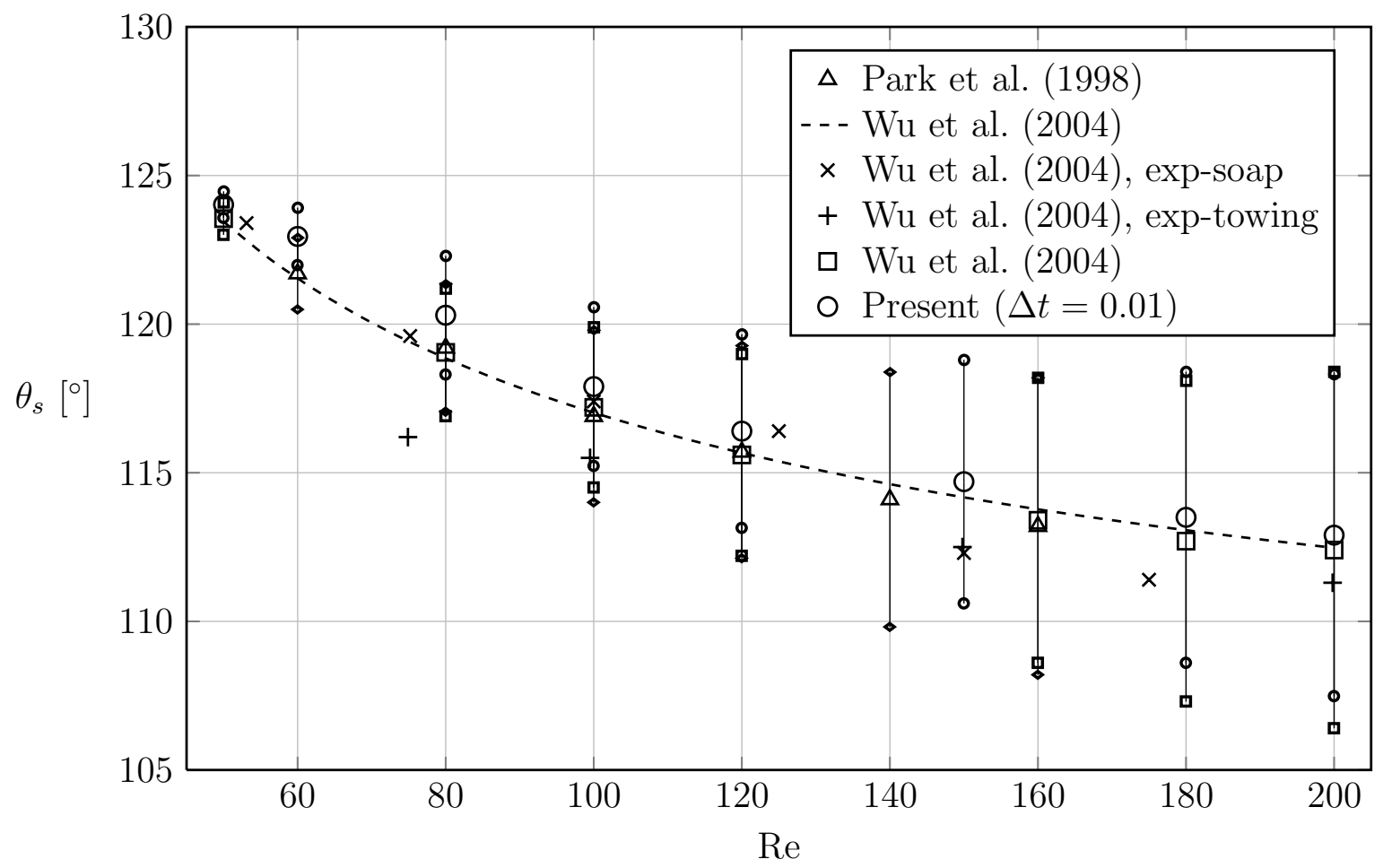

Figure 8: Separation angles versus Reynolds number. Large symbols: time-mean values; small symbols: amplitudes.

Finally in this section, the Reynolds number dependency of the time-mean suction pressure coefficients is shown in Figure 9. Present (2-D) results fit well with (i) the experiments of Eisenlohr (1990) and Williamson and Roshko (1990), at least up to Re $\simeq 165$ where 3-D effects appear, and (ii) the gathered data of previous 2-D simulations, especially with Posdziech and Grundmann (2007). 


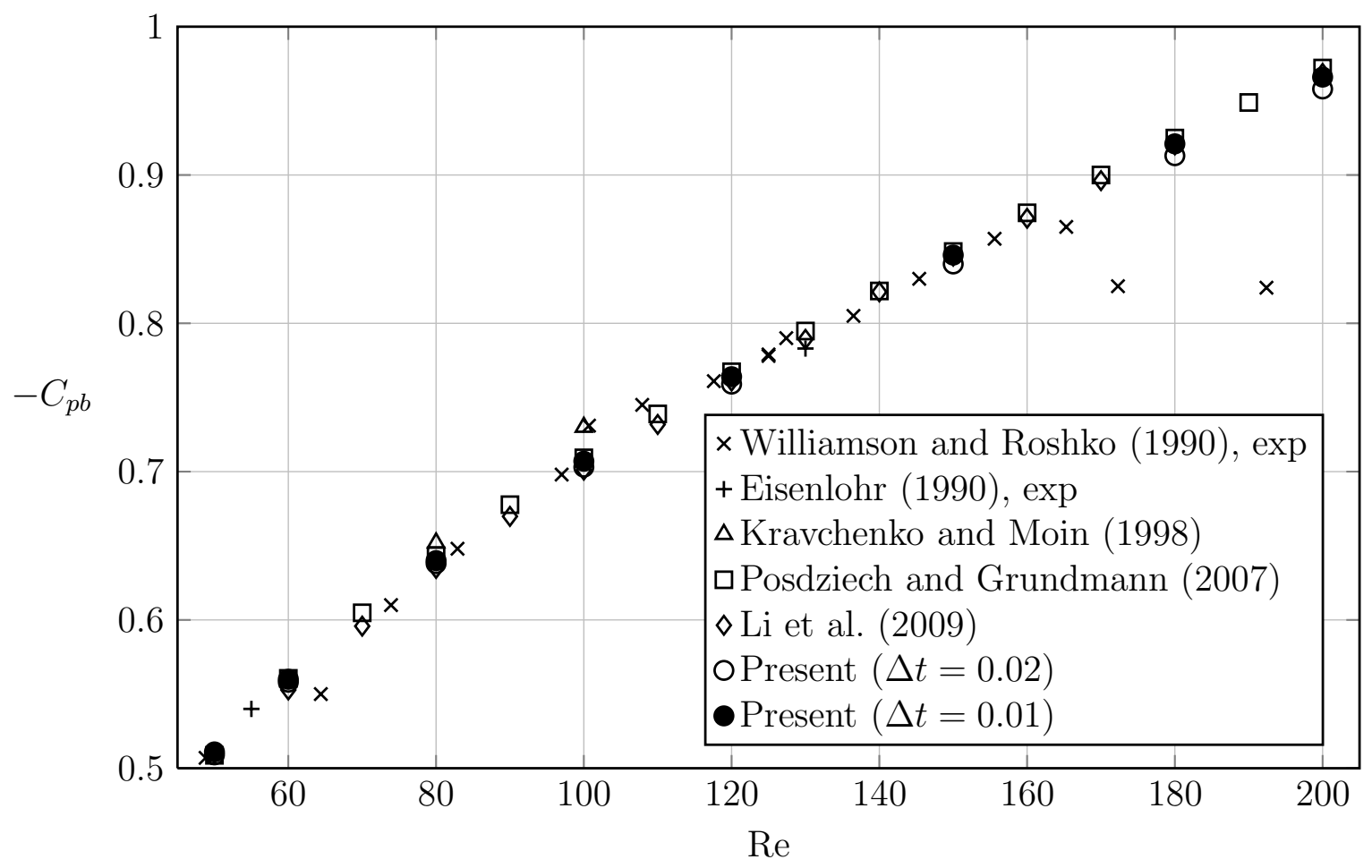

Figure 9: Base suction coefficient versus Reynolds number.

\subsubsection{Distributions along the wake center line and cylinder surface}

Some detailed comparisons for different Reynolds number are provided in the following figures, along the wake center line in Figure 10 and along the cylinder surface in Figure 11. Since the trends are monotonous with increasing Reynolds numbers, only results at $\operatorname{Re}=$ 50, 100, 150, 200 are displayed, for the sake of clarity.

Figure 10(a) depicts mean streamwise velocity distributions along the wake centerline. For Reynolds numbers higher than 50, there was a velocity reduction behavior in the region of $4.2<x<52$. When the Reynolds number was higher than 150 , the velocity reduction phenomenon became more complicated. In particular for $\mathrm{Re}=200$, the velocity decrease with increasing $x$ was already initiated at $x=4.2$ and lasted to around $x=32$, with a distinct plateau between that ranged from about $x=9$ to $x=16$ at a wake deficit velocity of $u_{d}=1-\bar{u}=0.38$, and a second slight plateau from about $x=25$ to $x=28$ at a wake deficit velocity of $u_{d}=1-\bar{u}=0.54$, see Figure 10(a,b). Most previous studies focus on 
the near wake region (up to around $x=10$ ), and only few previous investigation was found that covers a longer downstream wake distance. For instance, Persillon and Braza (1998) carried out $3-\mathrm{D}$ simulations at $\mathrm{Re}=200,300$ and provided the streamwise mean velocity along the wake center line up to $x=20$. They noticed that the variation of the streamwise mean velocity displays a strange behavior, similar to the distribution downstream towards $x=25$ presented in Lomtev et al. (1998) for $\mathrm{Re}=100$, both showing similar trends as in Figure 10(a). In particular, Persillon and Braza (1998) tried to compare with the velocity in the far wake calculated according to the assumption of similarity and some other classical theories, but got a completely different behavior. No further discussion of this behavior can be found in their paper. This wake phenomenon is discussed further in Section 4.3.

Figure 10(c) illustrates the level of r.m.s. streamwise velocity fluctuations, $u^{\prime}$, along $y=0$. It can be seen that the peak fluctuations appear right behind the wake bubble (wake closure points are marked by " $\times$ "), ranging from $1.3<x<8.5$. The reason for this behavior is probably that the flow contracts and accelerates behind the bubble closure point, with the result of stronger velocity and pressure fluctuations. Corresponding peak values appear in distributions of $v^{\prime}$ and $C_{p^{\prime}}$ along $y=0$ as well, not exactly the same location but near, especially at higher Reynolds number cases. It can be noted that there is a second peak of $u^{\prime}$ for $\operatorname{Re}>150$, which occurs downstream of the initial decrease in $\bar{u}$, as shown in Figure 10(a,b).

Figure 10(e) shows the variation of time-mean pressure coefficient $C_{p}$ along $y=0$. The pressure coefficients at the rear decrease with an increase of Re, and all the distributions then decrease along $y=0$ until they reach a minimum value inside the wake bubble. The magnitude of the minimum values increases with Re in the near wake region; however, the location moves upstream both at lower Reynolds number $(\operatorname{Re}<100)$ and higher Reynolds number $(\operatorname{Re}>120)$. Afterwards, the pressure coefficients increase rapidly before $x \simeq 3$ and then steadily recover to the free stream value $\left(C_{p}=0\right)$. The r.m.s. pressure coefficients shown in Figure 10(f) reach a maximum value in the near wake region, right behind the wake bubble. The second peak that occurs in the region of $3.6<x<4.2$ exists at all Reynolds numbers higher than 80. The peak values increase with Reynolds number, with the 
location moving upstream at higher Reynolds number. Obviously, the pressure fluctuations get stronger and more complicated at higher Reynolds number.

Comparing the locations of the peak values of $u^{\prime}, v^{\prime}, C_{p}, C_{p^{\prime}}$ shown in Figure 10(c)-(f) and the corresponding wake closure points marked by " $\times$ ", it can be observed that only for $\operatorname{Re}=200$ are the peak values of $u^{\prime}, C_{p^{\prime}}$ located at the same place as the wake closure point, whereas the deviations get smaller with an increase of the Reynolds number. In addition, it is of interest to note that the peak values of $v^{\prime}$ are located downstream of the wake closure points for all Reynolds number cases, but the interval gap is smaller at a higher Reynolds number. Meanwhile, the values of $v^{\prime}$ are quite a bit larger than the other fluctuation quantities, mainly due to the oscillating shedding vortex movement. For $\operatorname{Re}=100$, the maximum $v^{\prime}$ appears at the same place as the maximum $u^{\prime}$, at around $x=2.9$, whereas, for $\operatorname{Re}>100$, the maximum $v^{\prime}$ is located downstream of the maximum $u^{\prime}$, with a deviation increasing with Reynolds number; for $\operatorname{Re}<100$, the maximum $v^{\prime}$ is located slightly upstream of the maximum $u^{\prime}$, with deviations less than $2.5 \%$. However, the case of $\operatorname{Re}=50$ is the exception, for which the maximum $v^{\prime}(x=8.9)$ is located downstream of the maximum $u^{\prime}(x=8.5)$. 


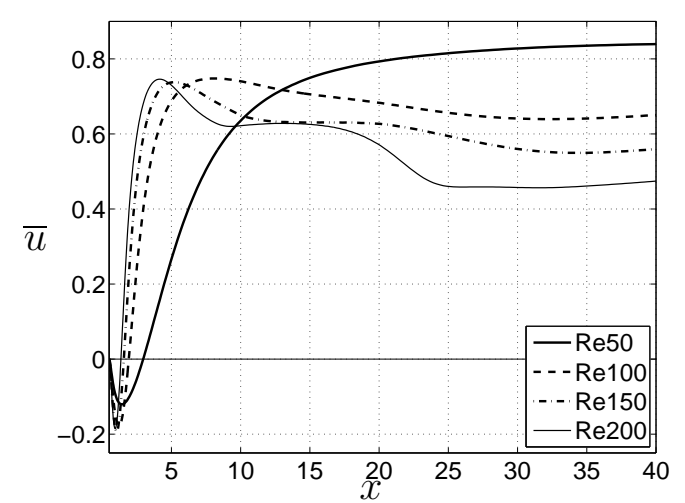

(a)

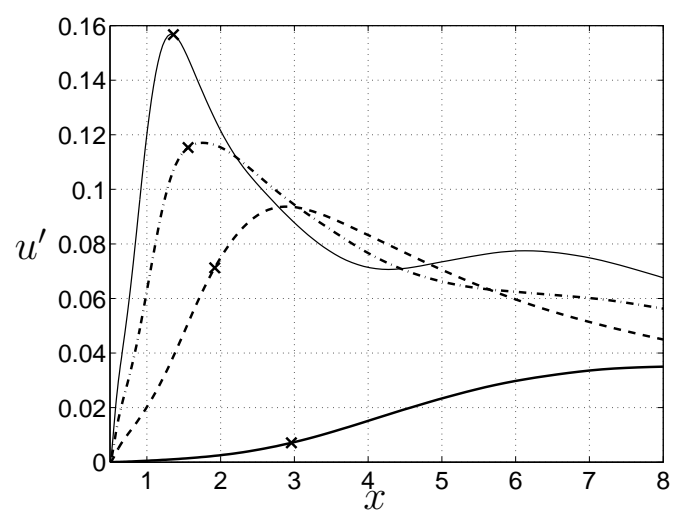

(c)

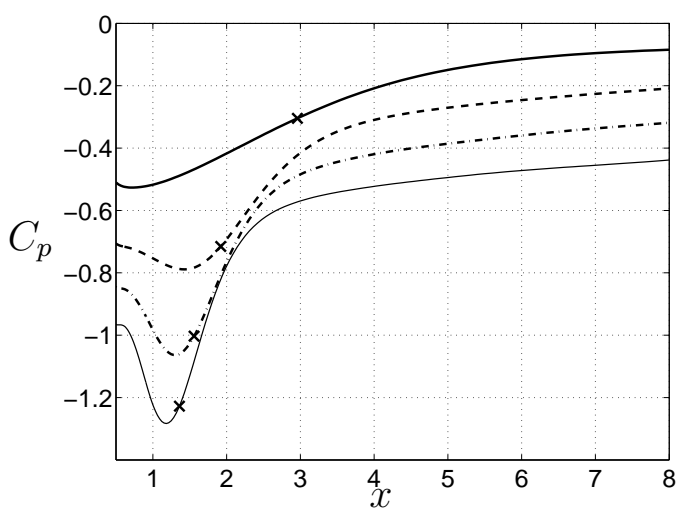

(e)

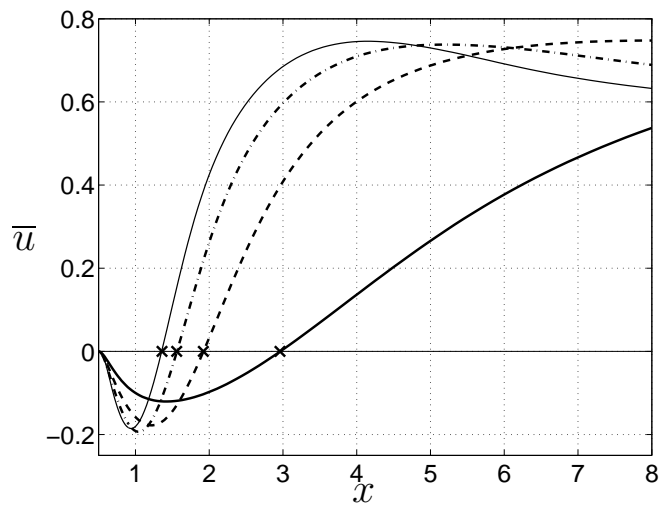

(b)

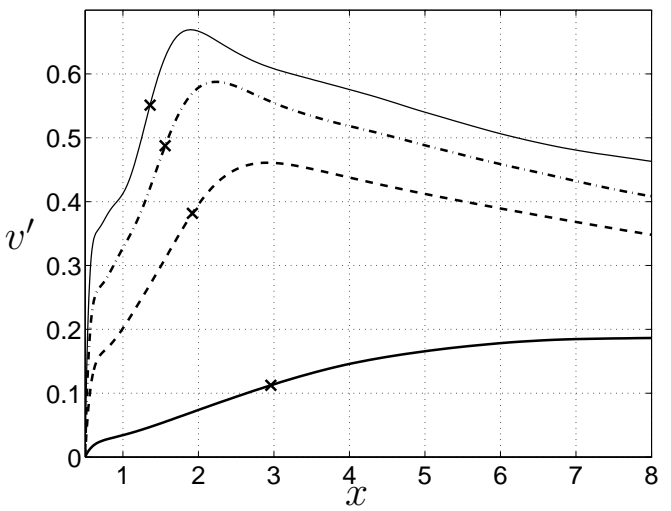

(d)

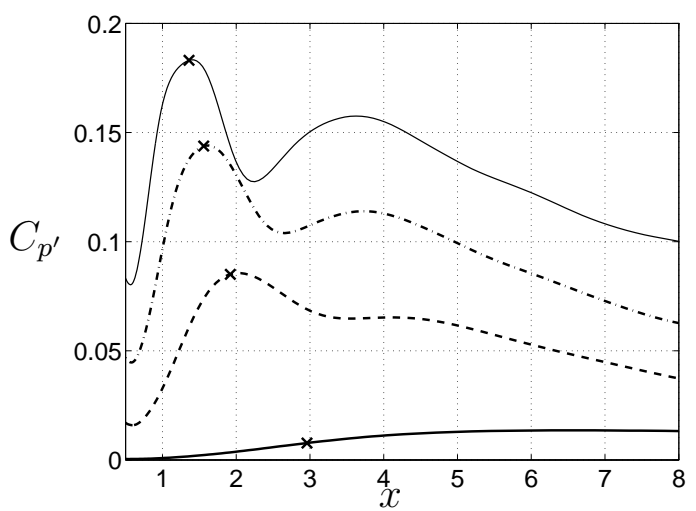

(f)

Figure 10: Comparison of flow along $y=0$ for $\operatorname{Re}=50,100,150,200$ (time-averaged wake closure points marked by $\times$ ): (a) mean streamwise velocity; (b) mean streamwise velocity in the very near wake region; (c) r.m.s. of mean streamwise velocity; (d) r.m.s. of mean vertical velocity; (e) pressure coefficients; (f) r.m.s. of the pressure coefficients. 
Figure 11(a) shows distributions of the time-mean pressure coefficient around the cylinder surface. It can be observed that the pressure coefficient at the rear of the cylinder $\left(C_{p b}\right)$ is more sensitive than at the front $\left(C_{p s}\right)$. Both decrease with increasing Reynolds number. A minimum is reached for the pressure coefficient in the range of $\theta=81^{\circ}-87^{\circ}$, showing a gradual upstream movement with increasing Re. Meanwhile, the corresponding locations of $C_{p}=0$ have the same upstream moving trends, for which a constant angular distance away from the minimum $C_{p}$ can be observed, with $\Delta \theta=(45 \pm 0.3)^{\circ}$. As can be seen in Norberg (2002), $C_{p}=0$ for cases in the subcritical regime, i.e. Re $>300$, almost located at the same angular positions $\left(\approx 35^{\circ}\right)$, albeit with a slightly decreasing trend with increasing Re, whereas, for the present laminar shedding regime, a maximum difference of $14 \%$ can be observed $\left(43.1^{\circ}\right.$ at $\operatorname{Re}=50,36.2^{\circ}$ at $\left.\operatorname{Re}=200\right)$.

The r.m.s. pressure coefficients are shown in Figure 11(b), where $C_{p^{\prime}}$ changes significantly with Re. The higher the Reynolds number, the stronger the fluctuations on the cylinder surface. As expected from a uniform and steady upstream flow, an extremely low level of $C_{p^{\prime}}$ at the frontal stagnation point $\left(\theta=0^{\circ}\right)$ is exhibited for all distributions. From this low stagnation value, there is a steady increase in $C_{p^{\prime}}$ up to a local maximum, which occurs at the angular position around $100.8^{\circ}$ for $\operatorname{Re}=50,97.0^{\circ}$ for $\operatorname{Re}=100,97.0^{\circ}$ for $\operatorname{Re}=150$ and $96.1^{\circ}$ for $\mathrm{Re}=200$. It can be seen that those peak values appear slightly upstream of the point where there is a local maximum absolute value for the mean pressure gradient, which can be detected in Figure $11(\mathrm{c})$, at around $101^{\circ}-110^{\circ} \cdot C_{p^{\prime}}$ then decreases slightly until around the angular position at around $123^{\circ}-133^{\circ}$, which can be classified as a second peak. Obviously, the pressure acting on the cylinder fluctuates more strongly on the sides than on the front and the rear, which explains why $C_{L^{\prime}}$ is so much larger than $C_{D^{\prime}}$.

Figure 11(c) shows (the negative of) the derivative of $C_{p}$, along the cylinder surface, $-d C_{p} / d \theta$, with $\theta$ in degrees. The absolute maximum pressure gradient occurs around $\theta=40^{\circ}$ $\left( \pm 0.2^{\circ}\right)$. All pressure gradient distributions then contract to one point at the angular position around $\theta=69^{\circ}$ and decrease rapidly to 0 . From the zero pressure gradient point, a positive pressure gradient occurs downstream towards the local absolute maximum value, at around $110.1^{\circ}$ for $\operatorname{Re}=50,104.5^{\circ}$ for $\operatorname{Re}=100,101.7^{\circ}$ for $\operatorname{Re}=150$ and $100.8^{\circ}$ for $\operatorname{Re}=200$. 
Afterwards, the flow separation points come up into the pressure gradient recovery region. A small negative pressure gradient region can be observed at $\operatorname{Re}>150$, which can be related to the distinct local maximum vorticity occurring around the base.

As mentioned in Norberg (2003), the position of local maximum for the r.m.s. pressure coefficient is an indicator of the mean separation point for high Re. The angular position of the local maximum for pressure gradient can also be used to indicate the mean separation point (Norberg, 2002), within the turbulent shedding regime. However, the related study of Sharman et al. (2005) indicates that, for $\mathrm{Re}=100$ within the laminar shedding regime, the actual separation occurs downstream of the positions detected by the above methods. This was further proven from the present study for $R e=50-200$, which can be seen from the points marked by " $\times$ " in Figure 11. From the collected maximum values above, it can be noticed that, for increasing Re within the laminar shedding regime, the angular difference between the separation point $\left(\omega_{z}=0\right)$ and the position of the local maximum for $d C_{p} / d \theta$ is $\left(14^{\circ}-12^{\circ}\right)$, while, with regard to the position of the local maximum for $C_{p^{\prime}}$, the difference is $\left(23^{\circ}-17^{\circ}\right)$. Further investigations are necessary to validate whether these angular positions may eventually merge at higher Re.

According to the boundary layer theory (Schlichting, 1979), as well as the more appropriate way to represent the relationships between the wake characteristics and the Reynolds number based on the square root of the Reynolds number (Williamson and Brown, 1998; Norberg, 2003; Wu et al., 2004), which is associated with the shear layer thickness in the laminar vortex shedding regime, the wall vorticity distribution was scaled with the square root of the Reynolds number and is shown in Figure 11(d). It can be seen that the higher Reynolds number has a stronger vorticity on the surface. The maximum value of vorticity appears at around $\theta=50^{\circ}$ for all Reynolds number cases, which shows good agreement with the values reported in (Park et al., 1998; Berthelsen and Faltinsen, 2008). Moreover, vorticity decreases monotonically downstream of the separation points for $\operatorname{Re}=50$, whereas a local maximum value can be observed at around $\theta=160^{\circ}$ for $\operatorname{Re}>100$. 


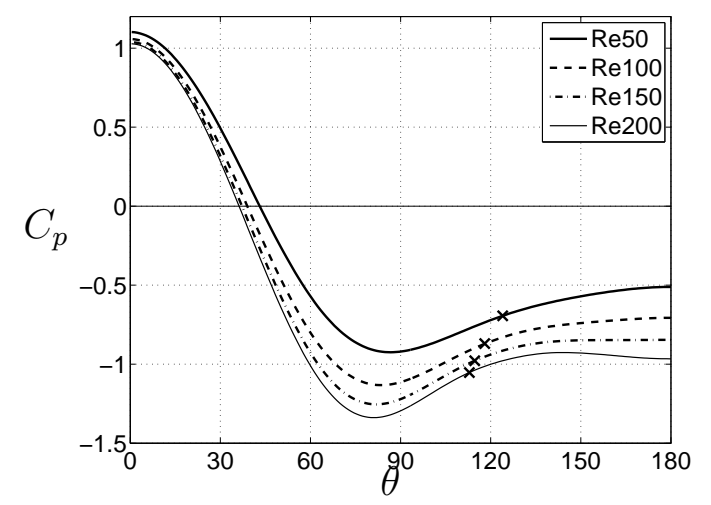

(a)

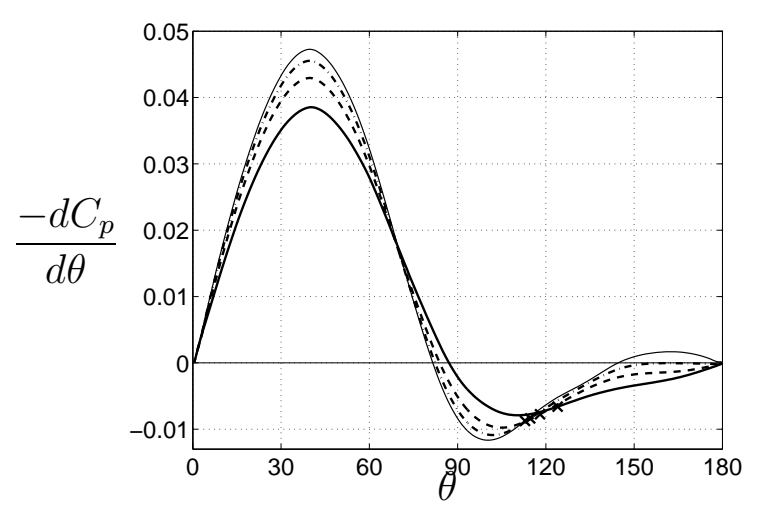

(c)

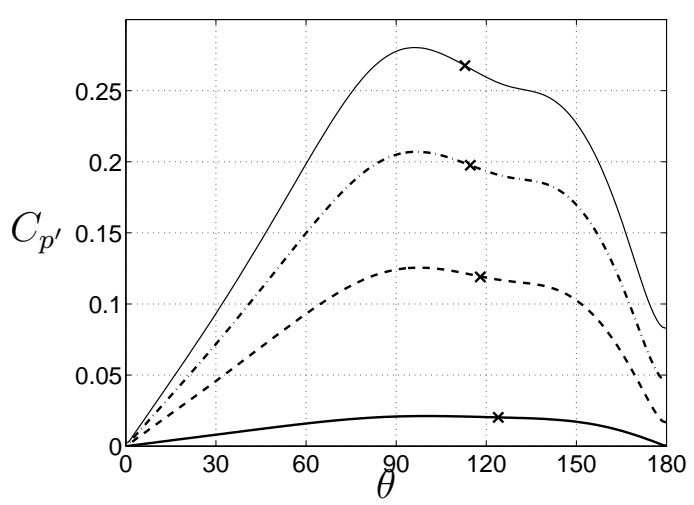

(b)

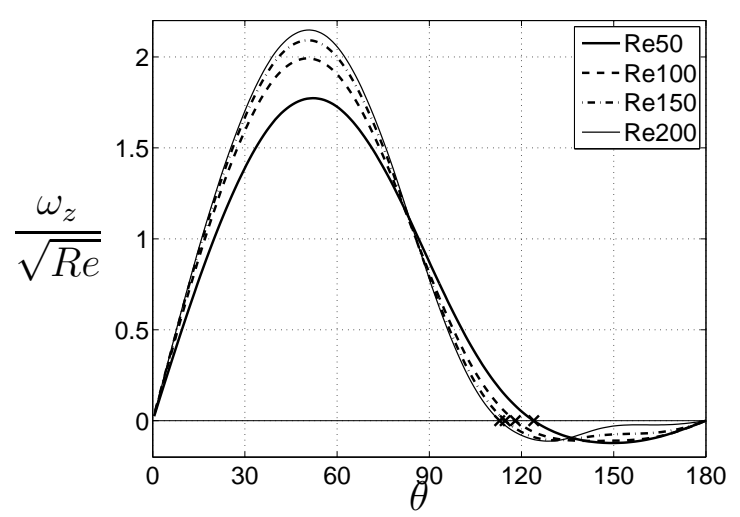

(d)

Figure 11: Mean (a), r.m.s. (b), gradient (c) of surface pressure and vorticity (d) distributions for $\mathrm{Re}=$ 50, 100, 150, 200 (time-averaged separation angles are marked by $\times$ ).

\subsection{Velocity deficit distributions along the wake center line $(\operatorname{Re}=150)$}

Regarding the wake flow of a circular cylinder, most studies focus on the near wake, presenting wake features and other results limited to the range of approximately $x<8$. Only a few previous investigations concentrate on the wake features and developments further downstream, even if it is known from experiments for laminar (near wake) shedding conditions that there exists a breakdown of the primary vortex street in the wake farther downstream with a subsequent development of a secondary vortex street (Cimbala, 1984; Williamson and Prasad, 1993; Karasudani and Funakoshi, 1994). For instance, at Re $=150$, the position for this breakdown is reported to occur within the range from about $x=50$ to $x=150$, the exact position being somewhat dependent on the experimental conditions and 
on the detection criteria; see Cimbala (1984), Williamson and Prasad (1993) and Karasudani and Funakoshi (1994). In Inoue and Yamazaki (1999), the breakdown has also been observed in 2-D numerical simulations.

In the present study, one interesting observation of the results along the wake centerline is that, for $\operatorname{Re}>50$, the streamwise velocity decreases in the wake region $x=4-52$ and then recovers towards the free stream velocity. As the Reynolds number gets higher, the velocity recovery becomes more complicated in this region. Regardless of whether if we change the domain size, mesh, time step or numerical scheme, this phenomenon always shows up. We also carried out similar simulations using different commercial codes, namely FLUENT, Star-CD and FLOWPHYS, and got similar results.

The present global results for $\mathrm{Re}=150$ are summarized in Table 3 (two cases: $H=$ 100,160). The case of $H=160$ was extended directly from $H=100$ by increasing the mesh cells, while keeping the inner grid the same. Figure 12 shows a comparison of the streamwise velocity deficit along the wake centerline for $R e=150$. There is a significant disagreement with the comparison experimental results (Cimbala, 1984; Williamson and Prasad, 1993), especially for $x<80$. Besides being fully 3-D, these experimental results, as evidenced in Williamson and Prasad (1993), are to some extent facility-dependent. However, the 2D results of Inoue and Yamazaki (1999) also show disagreement with our present data in the region of $x<80$. Even though a rather large blockage $(H=60)$ and short upstream distance $\left(X_{u}=30\right)$ were used in their study, a similar velocity deficit is expected to be observed. Besides, they used a quite large downstream distance $\left(X_{d}=400\right)$, although only with around 1841 grid points. Considering that a radius of 8 was adapted for the polar subdomain, the mesh beyond $x=8$ must then be a bit coarse. The numerical issues mentioned might be part of the reason that they did not capture the wake streamwise velocity deficit as we do. The loosely dotted line in Figure 12 refers to the far-field approximation behind a cylindrical body in two-dimensional steady flow, as adopted from White (1991). For the circular cylinder case, the deficit velocity along the wake center line can be written as

$$
u_{\mathrm{d}}=C_{D} \sqrt{\frac{\mathrm{Re}}{16 \pi\left(x-x_{0}\right)}},
$$


where $x_{0}$ can be seen as an effective position of the far wake generation, see Eames et al. (2011). With regard to the present 2 -D simulations at $\mathrm{Re}=150, C_{D}=1.30, x_{0}=25$ was adapted. As seen in Figure 12, $x_{0}=25$ fits the results rather well, but only from about $x=60$ and onwards.

Karasudani and Funakoshi (1994) experimentally studied the evolution of the vortex street and found that the primary vortex street breaks down into a nearly parallel shear flow with a Gaussian profile at a certain downstream distance, before a secondary vortex street of larger scale appears further downstream. They suggested that the viscous effect probably contributes to this breakdown process. Their discussion inspired us to look at the vortex evolution especially in the non-monotonous wake deficit region. We made several movie clips of the vortex development for $\operatorname{Re}=50,100,150,200$, covering the downstream region up to $x=40$. Similar to the results by Karasudani and Funakoshi (1994), the primary vortex street evolves into a nearly parallel shear flow for $\operatorname{Re}=100,150,200$. Moreover, the distortion and turning of von Kármán vortices can be observed twice for $\operatorname{Re}>50$, see Figure 13. For $R e=100,200$, shown in Figure 13(b,d), this behavior is in general agreement with the results of Mittal (2005). The first one appears around $x=5$, where the von Kármán vortices are initially formed and shed from the shear layer, with the vortex center quickly moving away from the wake centerline. This is probably the reason for the local maximum value observed in the streamwise velocity deficit, see Figure 10(a). The second one shows up at around $x=20$ and further downstream, which is especially apparent at higher Reynolds number, say $\operatorname{Re}=150,200$. As can be seen in Figure $13(\mathrm{c}),(\mathrm{d})$, beyond $x \approx 15$, the vortex centers, as indicated from local extreme values of $\omega_{z}$ within the vortices, were at an almost constant cross-stream distance from the wake center line. During the initial transferring process, there is a change-over in the tilting of the von Kármán vortices, and the shape of the vorticity region changes from being closely circular to kind of elliptical. Moreover, a merging trend can be seen for $\operatorname{Re}=200$ beyond $x=25$, where the neighboring von Kármán vortices seem to connect with each other. The issues mentioned above might be the reasons for the plateau-like behavior in the stream velocity deficit region, see Figure 10(a) and Figure $12(\operatorname{Re}=150)$. However, none of the above phenomena can be found for $\mathrm{Re}=50$ 
(Figure 13(a)), which explains why the streamwise velocity distribution along $y=0$ increases monotonously after the wake bubble for $\mathrm{Re}=50$. As providing further confidence on the velocity deficit behavior found in the present study, the decay of the peak vorticity within the von Kármán vortices was found to be in agreement with the numerical results reported in Ponta (2010) and the soap-film experimental results in Vorobieff et al. (2002).

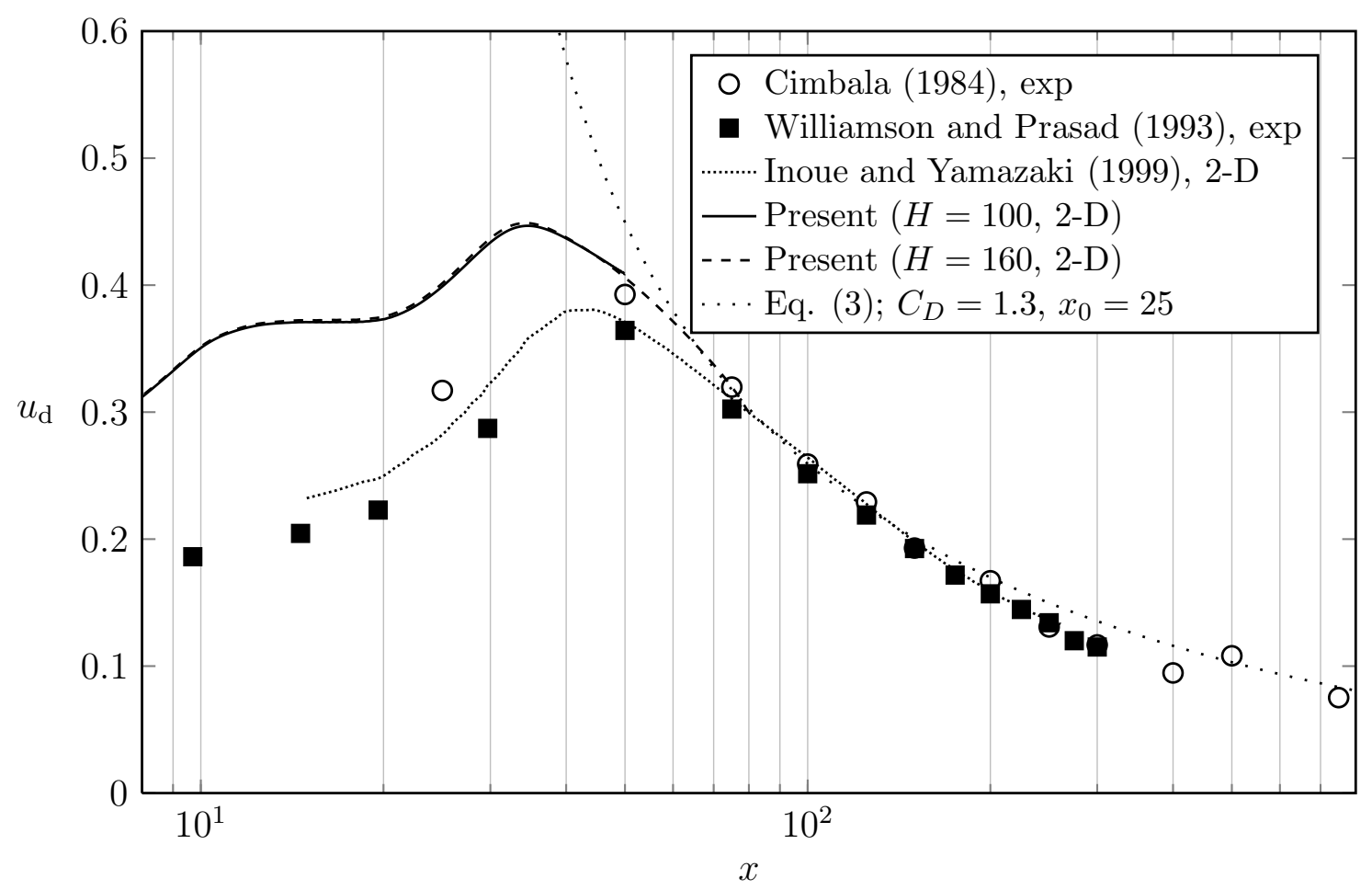

Figure 12: Streamwise velocity deficit along the wake centerline, $u_{\mathrm{d}}=1-\bar{u}(x, 0), \operatorname{Re}=150$.

\subsection{3-D simulations; $\operatorname{Re}=200$}

Experimentally, the highest Reynolds number for which a continuous laminar shedding flow can be maintained is about $\mathrm{Re}=190$ (Miller and Williamson, 1994; Williamson, 1995). For completeness, we therefore present in this subsection some results of 3-D simulations at $\operatorname{Re}=200$, using two spanwise lengths, $L_{z}=10$ and $L_{z}=12$.

\subsubsection{Preliminaries and global results}

For realizability of a 3-D simulation of this canonical flow case, the spanwise dimension of the cylinder, the spanwise length $L_{z}$, should be long enough to be able to capture the 


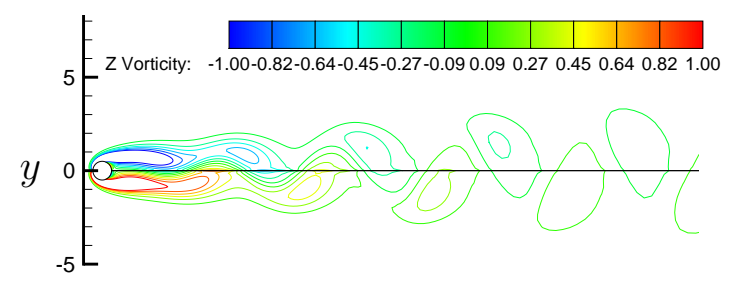

(a)

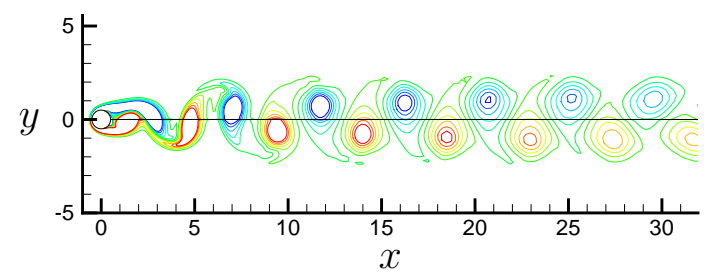

(c)

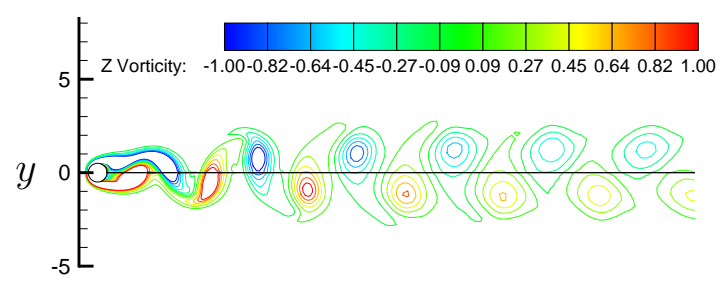

(b)

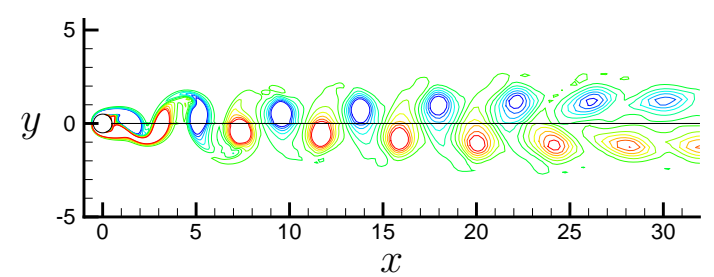

(d)

Figure 13: Comparison of instantaneous vorticity $\left(\omega_{z}\right)$ at different Reynolds numbers: (a) $\operatorname{Re}=50$; (b) $\operatorname{Re}=100 ;(\mathrm{c}) \operatorname{Re}=150 ;(\mathrm{d}) \operatorname{Re}=200$.

essentials of the anticipated large-scale 3-D spanwise flow developments (Gioria et al., 2011). For the same reason, it is also required to employ a spanwise resolution that is fine enough, as judged from the anticipated smallest flow features of dynamical importance, a requirement that also involves considerations of the approximation order of the employed numerical schemes. In addition, the spanwise boundary condition ought enforce a minimum amount of disturbance from the cylinder ends, and especially so when using a limited spanwise length.

The experiments of Norberg (2003) indicate that the one-sided spanwise correlation length of lift-related near wake velocity fluctuations in the inital transition regime (up to about $\operatorname{Re}=220$ ) is about 7 units. Moreover, when using end plates in a laboratory experiment, the study of Norberg (1994) shows that the Strouhal number and base suction coefficient at around $\mathrm{Re}=200$ are unaffected by the aspect ratio (length-to-diameter ratio) down to ratios of about 30. As the boundary conditions adopted at the cylinder ends (Section 2.2) are believed to be of a much softer nature than the above experimental ones with a no-slip termination, the choice of using a maximum spanwise length of about half the one required from experiments seems motivated. In addition, at $\operatorname{Re}=200$, the neutral curve of possible mode $\mathrm{A}$ wavelengths that in fact can be excited ranges from about $\lambda_{z}=3.3$ to 
$\lambda_{z}=5.1$, the most unstable being at $\lambda_{z}=3.9$ (Barkley and Henderson, 1996; Posdziech and Grundmann, 2001). This means that $L_{z}=12$ is more than twice the largest mode-A wavelength that can be excited and about three times the most unstable one. Due to computer limitations, it was not possible at this stage to carry out any tests with even longer spanwise lengths.

Some selected global results for the two 3-D cases and a corresponding 2-D case are given in Table 4. For a lateral domain width of $H=80$, the near wall and outlet wake resolution parameters were here set at $\delta=0.0035, \delta_{e}=0.21$, using sectional mesh $386 \times 322$. The time step was $\Delta t=0.02$. To indicate the lower expected accuracy when using this longer time step (Section 4.1.3), in combination with an integration time that for computer time reasons was limited to about 100 time-mean shedding periods, the number of significant digits in Table 4 have been limited to three. Both 3-D cases in Table 4 had about 13 spanwise grid points per unit diameter, corresponding to a spanwise distance between the equidistant planes of about $\Delta z=0.075$. When including all related investigations referred to in this part, Posdziech and Grundmann (2001); Carmo and Meneghini (2006); Labbé and Wilson (2007); Behara and Mittal (2009); Rajani et al. (2009); Cao et al. (2010); Leontini et al. (2010), the average number of spanwise points/nodes per unit diameter is approximately 10. Further, for $\operatorname{Re}=220$, it is demonstrated in Posdziech and Grundmann (2001) that about 8.5 spanwise points/nodes per unit diameter is sufficient to produce converged global results, at least for $\left(\mathrm{St}, C_{D}\right)$. In Carmo and Meneghini (2006) but for Re $=320$, the number of spanwise points/nodes to achieve an "adequate representation of the flow" was found to be approximately 12.8 spanwise nodes per unit diameter. From these facts the present spanwise resolution levels seem well motivated to be sufficient.

These above previous investigations all employed periodic spanwise boundary conditions, except Behara and Mittal (2009) who used the same spanwise boundary condition as in the present study, the symmetry condition. At least for relatively short spanwise lengths, it can be argued that the symmetry condition ought to be more physical than the spanwise periodic one; there is no particular reason why the flow should instantaneously repeat itself at specific spanwise positions. Here it should be noted that the values gathered in Table 4 
were calculated by taking away spanwise sectional values at distances 0.3 units away from each end of the cylinder, thus reducing possible end condition effects from the forcing of vanishing spanwise velocity at the ends (Section 2.2). Please note that the $C_{L^{\prime}}$ and implied $C_{D^{\prime}}=C_{L^{\prime}} / r$ in Table 4 represent spanwise-averaged sectional values.

Table 4: Global results for $\operatorname{Re}=200, H=80, \Delta t=0.02$.

\begin{tabular}{cccccccc}
\hline Case & $C_{D}$ & $C_{D p}$ & $r$ & $C_{L^{\prime}}$ & $-C_{p b}$ & $L_{c}$ & St \\
\hline 2-D & 1.32 & 1.07 & 15.9 & 0.457 & 0.963 & 0.88 & 0.196 \\
3-D, $L_{z}=10$ & 1.24 & 1.00 & 12.1 & 0.339 & 0.847 & 1.15 & 0.181 \\
3-D, $L_{z}=12$ & 1.25 & 1.01 & 8.31 & 0.350 & 0.861 & 1.11 & 0.183 \\
\hline
\end{tabular}

As mentioned earlier (subsection 2.2), the cases with $L_{z}=10$ and $L_{z}=12$ eventually went into a state that can be characterized as a spatiotemporal chaotic flow (Henderson, 1997), in laboratory experiments denoted as mode $A^{*}$ (Williamson, 1996a); see Figure 14 and Figure 17. Although both these cases produced global results in reasonable agreement with available experiments $\left(C_{D},-C_{p b}\right.$, St), the case with $L_{z}=10$ exhibited features that indicated a strong influence from the its spanwise length. For instance, the spanwise correlation coefficient of fluctuating sectional lift ( $R_{L L}$, not shown) for $L_{z}=12$ exhibited an expected monotonous drop-off appearance at large separations, almost reaching zero correlation $\left(R_{L L} \simeq 0.05\right)$ at a spanwise separation distance of 10.5 length units, similar in appearance with the lift-related correlation function as presented in Norberg (2003) for $\operatorname{Re}=240$ but in sharp contrast to the one for $L_{z}=10$ that exhibited a seemingly non-physical appearance at separation distances greater than about 2 (units), showing undulations on a declining trend but with all correlation levels above $R_{L L}=0.65$. When calculating these correlation functions, the reference plane was placed at about 0.5 units from either end of the cylinder. Since the case with $L_{z}=10$ exhibited non-physical features albeit producing seemingly reasonable global results, all aspects as provided below, except when specifically noted, are related to the case with $L_{z}=12$. 


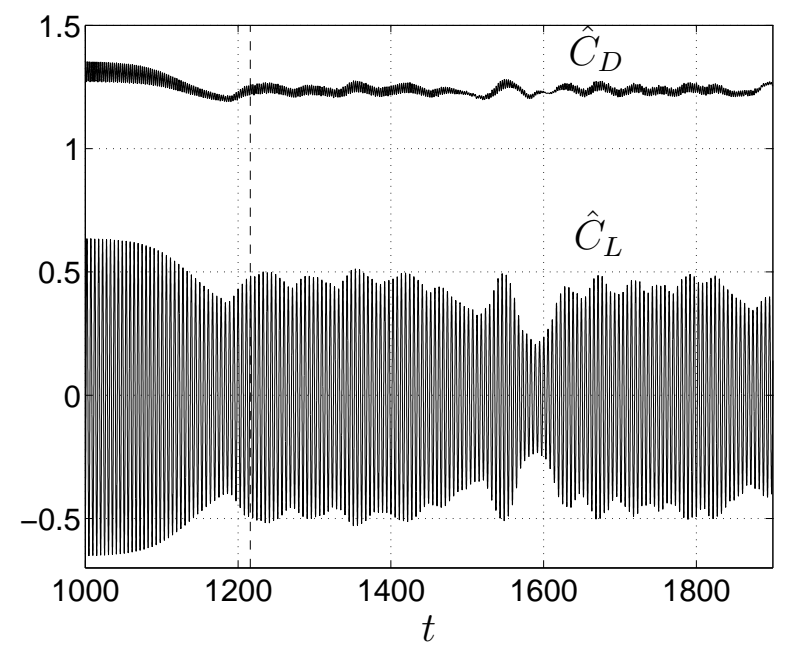

(a)

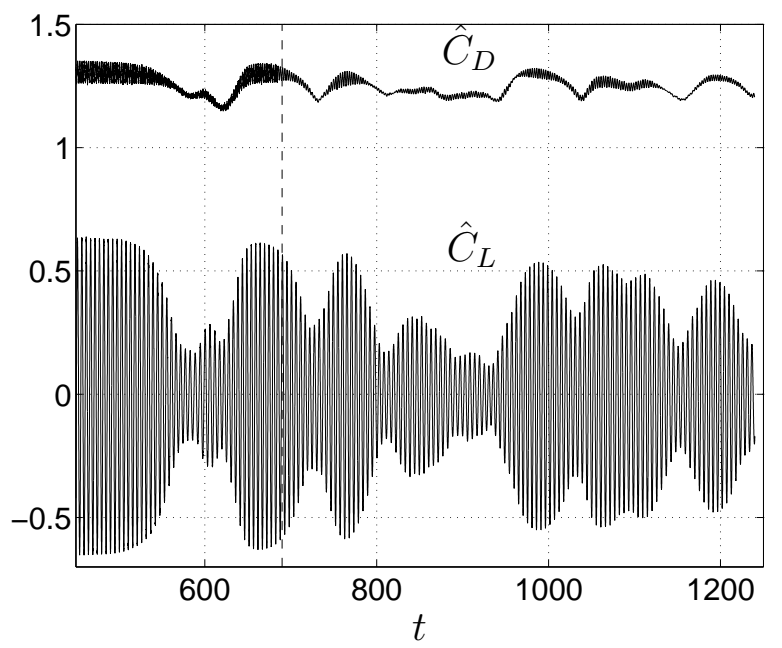

(b)

Figure 14: Time history of spanwise-averaged lift and drag coefficients for $\operatorname{Re}=200$ : (a) $L_{z}=10$, (b) $L_{z}=12$. The dash lines indicate the start time for integrations.

\subsubsection{Comparisons with 2-D, experiments and previous 3-D studies}

The base suction coefficient from the 3 -D simulation with $L_{z}=12\left(-C_{p b}=0.861\right)$ is within $\pm 2.5 \%$ when compared with the experimental results $\left(-C_{p b} \simeq 0.84-0.88\right)$ of (Williamson and Roshko, 1990; Norberg, 1994, 2002). The corresponding Strouhal number of $\mathrm{St}=0.184$ is within the range of $\mathrm{St}=0.182-0.184$, which represents values reported in (Norberg, 1987; Williamson, 1988; Norberg, 1994; Fey et al., 1998). The pressure drag coefficient of $C_{D p}=1.01$ is in close agreement with the experimental value of $C_{D p}=1.02$ reported in Norberg (2002), see also Figure 15(a).

In previous numerical studies for $R e=200$, Posdziech and Grundmann (2001) reported St $=0.182, C_{D}=1.24$ and $-C_{p b}=0.87$ from a simulation with $H=140$ and a spanwise periodic length equal to the most unstable wavelength of the mode A instability, $L_{z}=3.9$, and about 8.3 spanwise spectral nodes/points per unit diameter. The results of Posdziech and Grundmann (2001) are intriguing. As demonstrated convincingly in Henderson (1997), the expected scenario when using a spanwise length matching the most unstable mode A wavelength, in a regime where this is the only 3-D instability that can be excited, the flow ought to be locked to a deterministic and time-periodic state with only clean mode A struc- 
tures present. In Posdziech and Grundmann (2001) the indicated final state is much more disordered, displaying strong and seemingly random variations in the temporal development of the indicated Strouhal number. The reasons for this behavior are unclear. They did not test other spanwise lengths since the results fitted very well with experimental data. Carmo and Meneghini (2006) carried out tests with spanwise periodic lengths varying from $L_{z}=3$ to $L_{z}=18$ using (at least) 12.8 spanwise spectral nodes per unit diameter and $H=100$. For $L_{z}=18$ they reported $\mathrm{St}=0.185$ and $C_{D}=1.27$; for $L_{z}=12$, St $=0.184$ and $C_{D}=1.28$. Labbé and Wilson (2007), using $L_{z}=6.3$ and 20.4 spanwise spectral nodes per unit diameter, reported $\mathrm{St}=0.195, C_{D}=1.318$; these values are close to the ones reported in a corresponding 2-D simulation using the same cross-sectional polar grid/domain $(D=30)$ and are also in agreement with the 2-D results in Table 4 . The corresponding global results of Rajani et al. (2009), also using $L_{z}=6.3$ but at a significantly lower spanwise resolution (about 5.1 spanwise nodes per unit diameter), are very similar to the ones of Labbé and Wilson (2007); St $=0.1936, C_{D}=1.338$. The plot of vorticity fields in Labbé and Wilson (2007) for $L_{z}=6.3$ show a very regular pattern of mode A structures, which means that the "chaotic" state was not reached in this simulation. Interestingly, the change-over to the "chaotic" state is in Carmo and Meneghini (2006) reported to occur when the periodic length changes from $L_{z}=8$ to $L_{z}=12$, and being associated with a sudden drop in Strouhal number (from $\mathrm{St}=0.195$ to $\mathrm{St}=0.184$ ). Cao et al. (2010) reported $\mathrm{St}=0.186$ and $C_{D}=1.30$, using a polar sectional domain with $D=60, L_{z}=8$, and 10 spanwise grid points per unit diameter. For this simulation, in contrast to the results of Carmo and Meneghini (2006) for the same $L_{z}$, the final state appears to be the chaotic one, as judged from their vorticity plot $(\mathrm{Re}=200)$, and also manifested from their its' lower Strouhal number. Behara and Mittal (2009) reported St $=0.195, C_{D}=1.38$ using $L_{z}=10$ with 5.1 nodes per unit diameter. The flow state was associated with parallel shedding without any signs of vortex dislocations. Their vorticity plot do not suggest the presence of chaotic flow developments. However, it seems that only 120 time units were simulated. Based on the indeed very long time to reach the chaotic state for the matching case of the present study, it seems that insufficient simulation time is the plausible main cause of the deviations between the present results 
and those of Behara and Mittal (2009). Finally, Leontini et al. (2010) obtained St $=0.187$ and $L_{c}=1.07$, with $H=30, L_{z}=16$ and 6 spanwise spectral nodes per unit diameter. When considering the diversity in other numerical parameters as well, the present 3-D results (Table 4) are in reasonable agreement with the above numerical studies.

When comparing the $3-\mathrm{D}$ case of $L_{z}=12$ with the $2-\mathrm{D}$ case, the most severe effect can be found for the ratio $r$ between sectional r.m.s. drag and lift, which showed a reduction of $48 \%$; the reduction in the sectional r.m.s. drag coefficient, $C_{D^{\prime}}=C_{L^{\prime}} / r$, was $46 \% ; C_{L^{\prime}}$ decreased by about 23\%. Despite being quite comparable on Strouhal number, base suction, time-mean drag coefficients and sectional r.m.s. lift, respectively, there was a notable and significant difference between the two 3-D cases for the sectional r.m.s. drag; the sectional $C_{D^{\prime}}$ for $L_{z}=12$ was about $150 \%$ higher than for $L_{z}=10$ whereas the sectional r.m.s. lift only was about $3 \%$ higher. As discussed further in the next subsection, the reason for this large difference in sectional r.m.s. drag is that the case for $L_{z}=12$ exhibited distinct and occasional strong flow pulsations due to severe distortions of the shedding flow in the near wake region; for the case with $L_{z}=10$ such pulsations were also present but not as distinct and powerful as for $L_{z}=12$.

When compared with 2-D, the was a drop in Strouhal number of about 6\%. The Strouhal number within the long-lived intermediate 3 -D state with very weak mode A instability structures, in Figure 17(a) referred to as the "quasi-2D" state, only was about $1.5 \%$ lower than the corresponding 2-D value. This successive decrease in Strouhal number is in general agreement with the scenario presented in Henderson (1997) for $\operatorname{Re}=220$ and $L_{z}=14.78$.

The one-sided correlation length of sectional lift fluctuations, calculated from integration of the cross-correlation function $R_{L L}$ (as briefly described in Section 4.4.1), was about 5.1 units. The ratio $\gamma_{L}$ between spanwise-averaged to spanwise-mean sectional r.m.s. lift was calculated to $\gamma_{L}=0.784$, in agreement with the value calculated from the formula for $\gamma_{L}$ in Norberg (2003) using the correlation function $R_{L L}$ and a segment length equal to the the spanwise length, $L_{z}=12$. Since the reference position for $R_{L L}$ was placed at about 0.5 units from either end of the cylinder, the remaining small part (up to a separation corresponding to the full cylinder length) was simply extrapolated to $R_{L L}=0$. From this exercise it can 
be deduced that the effects from the symmetry condition enforced at the cylinder ends were indeed very small. This was also inferred from the very small differences between spanwiseaveraged sectional time-averaged values using the full spanwise length and the time-averages of spanwise-averaged values of quantities that for spanwise homogeneous conditions should not depend on the segment length of integration, e.g. St and $C_{D}$.

Finally, when comparing with the experimental results on the time-averaged $C_{p}$-distribution of Norberg (2002), see Figure 15, the 3-D results show good agreement. It can be seen that the deviation of $C_{p}$ between the 2-D and the 3-D results, as well as the experimental data, occurs at around $50^{\circ}$ and gets larger in the downstream direction, where the unsteady 3-D effects appear. However, the pressure coefficients for the 3-D case fluctuate more weakly on the cylinder sides, comparing with the 2-D results, see Figure $15(\mathrm{~b})$. This is related to the drop of the r.m.s. lift coefficient.

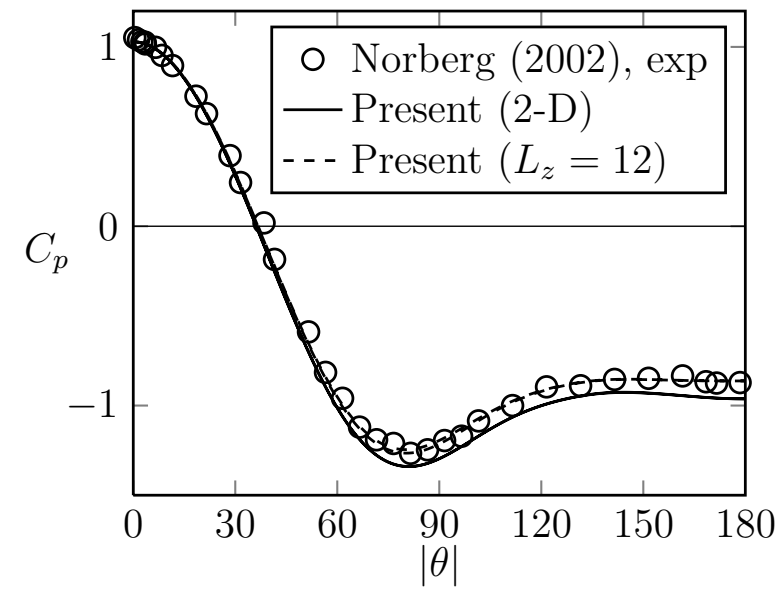

(a)

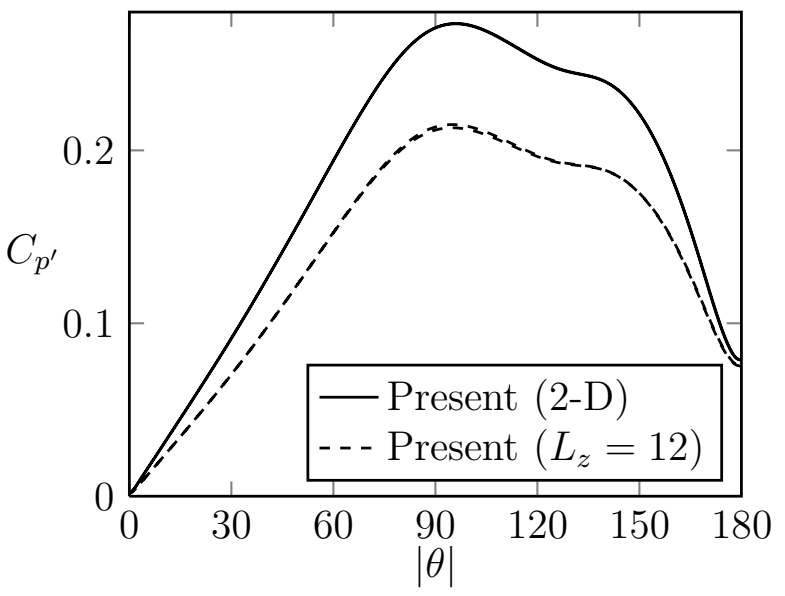

(b)

Figure 15: Pressure coefficients distributions along the cylinder wall, $R e=200$.

\subsection{3. $L F$ and $H F$ regions}

A distinct pulsation can be observed from the time history of force coefficients shown in Figure 14, indicating high and low levels of forces, which can be referred to as HF and LF regions (Sohankar et al., 1999). Similar force pulsations were observed by Sohankar et al. (1999) for square cylinder flow, while no numerical investigations regarding circular cylinder 
flow have yet been found. As evident in Figure 14(a), the envelope of force fluctuating amplitudes within HF regions is close to the level within the initial state (approx. $60<$ $t<500)$, a state in which the wake flow exhibited rather weak but clearly distinguishable and regular mode A instability structures, in three pairs, indicating, as expected, a most unstable wavelength of about 4 diameters (Posdziech and Grundmann, 2001). Within the HF regions, the spanwise near wake flow also exhibited those mode A instability structures, but not as regular as in the initial region. Within the time intervals of low force amplitudes, the LF regions, the near wake flow was much more chaotic, albeit with occasional remnants of mode A. Overall, it seems that the wake flow from about $t=550$ and onwards can be characterized as being of mode $\mathrm{A}^{*}$, as described in Williamson (1992).

Regarding the above two distinct pulsation regions, a significant spanwise variation of sectional lift and drag coefficient versus time in Figure 16 can be proposed as evidence, associated with the three-dimensionality development. It can be seen in Figure 16(a) that the lift fluctuations are essentially in phase over the spanwise length, except for occasional events of the extreme LF regions that occurred at around $(z=2.0, t=729),(z=-0.60, t=808)$, $(z=-4.6, t=836),(z=-5.5, t=879),(z=0.45, t=927)$ and $(z=-1.5, t=939)$. At the location for the first-in-time of these LF regions, which is the most extreme one in this plot, the minimum sectional drag coefficient value was as low as 1.07 , about $14 \%$ lower than the time- and spanwise-averaged value $\left(C_{D}=1.25\right)$. The corresponding spanwise-averaged envelope drag coefficient at this time (Figure $14(\mathrm{~b})$ ) was about $\hat{C}_{D}=1.20$, about $4 \%$ lower than $C_{D}=1.25$. The sectional lift amplitude at around this most extreme LF region is almost zero, which is much lower than the time-corresponding spanwise-averaged envelope lift coefficient of $\hat{C}_{L}=0.30$ from Figure 14(a). As for the forces acting on the cylinder and at this particular Re, the LF regions are thus indicated to be rather spanwise localized with small to moderate effects on the spanwise-averaged force at the particular time of its occurrence.

Within time period of Figure 16, which covers about 52 time-mean shedding periods, two events of near-wake spanwise vortex dislocations were observed. The actual dislocation behavior was observed from movie clips on the evolution of spanwise vorticity but can also 
be inferred from the Y-shaped connections of the iso-contours of sectional lift at the right end of Figure 16(a). The flow features in the near wake region with regard to different flow states, "quasi-2D" state $(t=463)$, "chaotic" state with LF $(t=928)$ and HF $(t=974)$ region, are illustrated in Figure 17. Obviously, quite regular vortex structures can be observed at $t=463$, whereas a major vortex dislocation is present at $t=928$ (LF region). No vortex dislocation events in the very near wake were observed for the case with $L_{z}=10$. At worst, there was a significant phase distortion at around the time for the lowest envelope amplitude of the lift fluctuations that occurred at around $t=1600$, see Figure 14(a). For a more complete picture of how the LF-regions and these seemingly rare occasions of vortex dislocations are distributed both in time and spanwise requires studying longer time intervals and longer spanwise dimensions. That was not possible within the limitations of this study. 


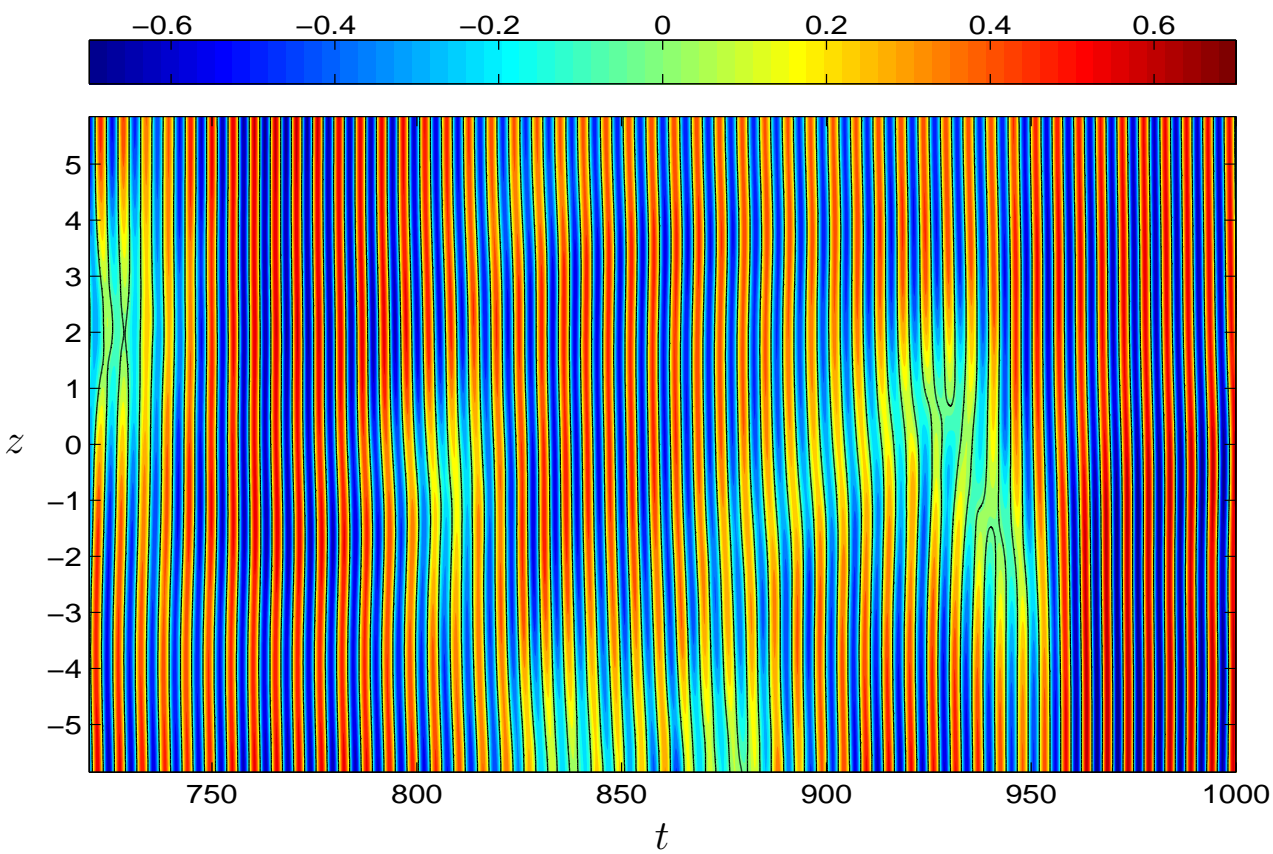

(a)

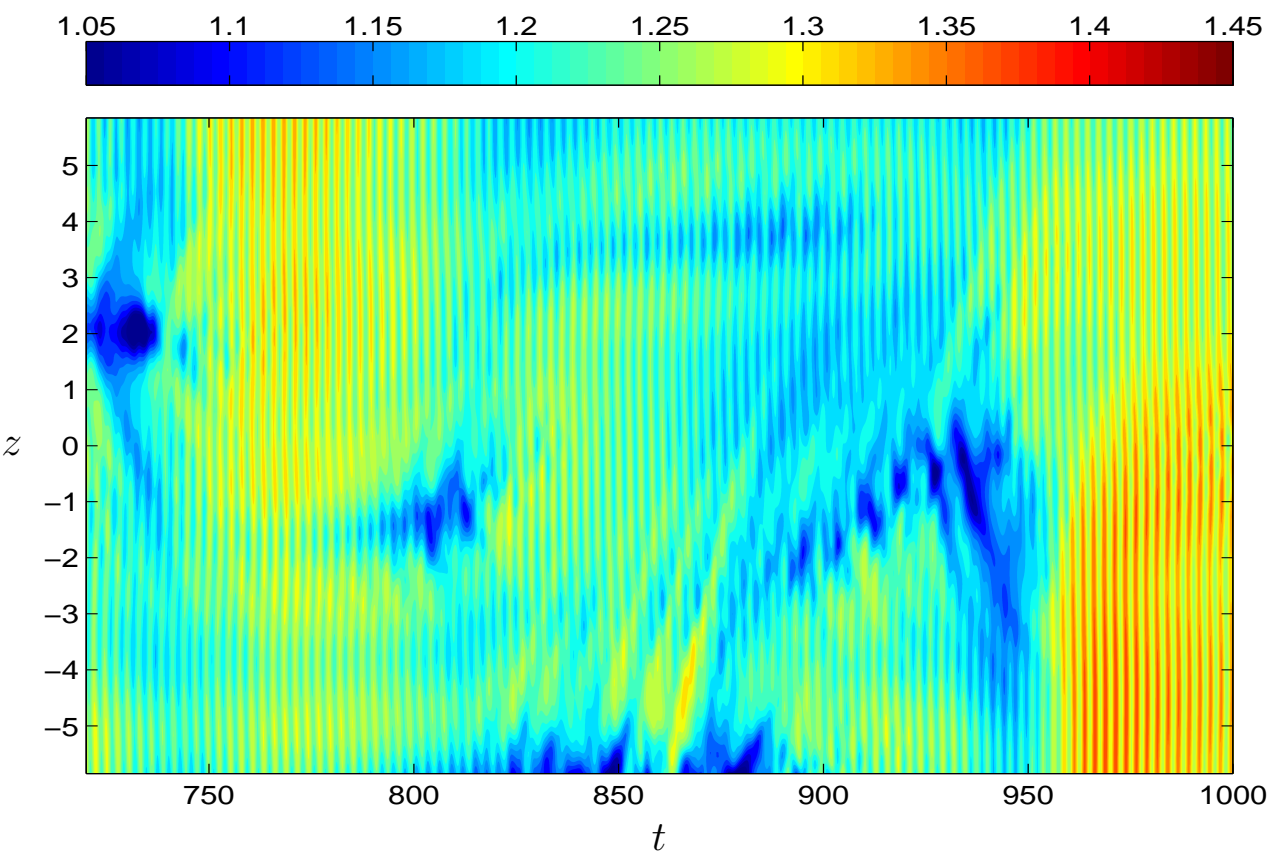

(b)

Figure 16: Isocontours of sectional lift (a) and drag (b) coefficients versus time and the spanwise position for $\operatorname{Re}=200\left(L_{z}=12\right)$. The black lines in (a) indicate zero values of sectional lift. 

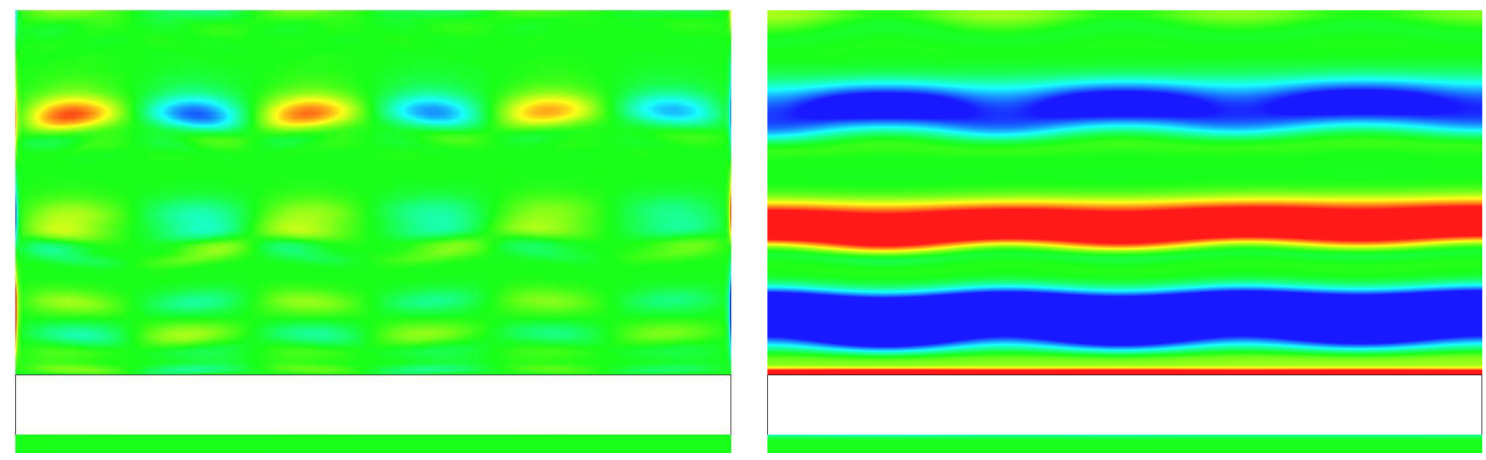

(a)
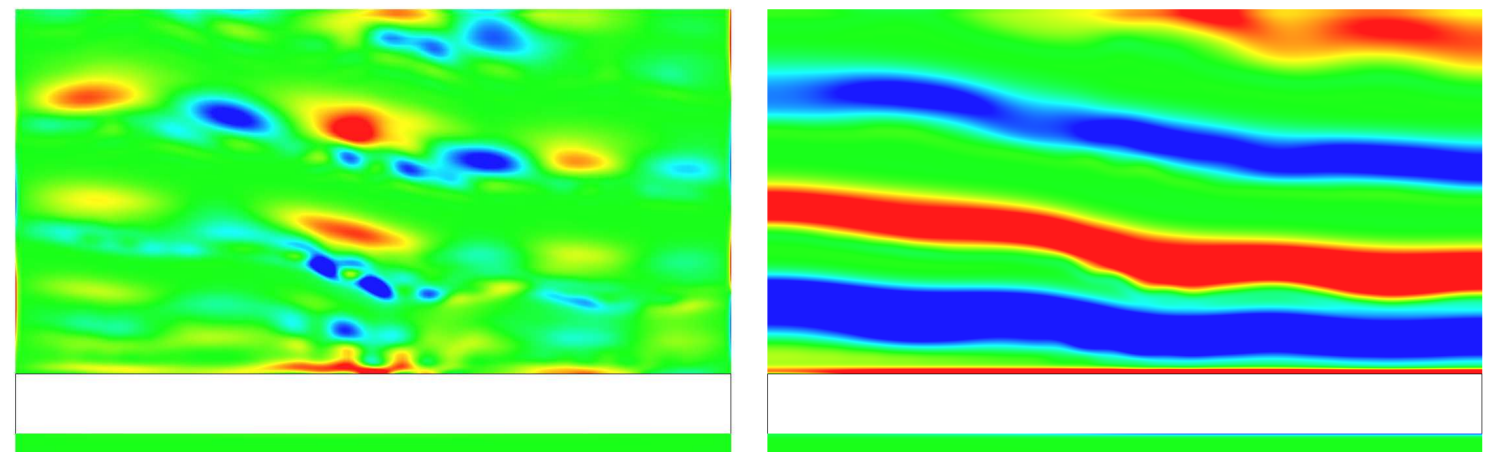

(b)
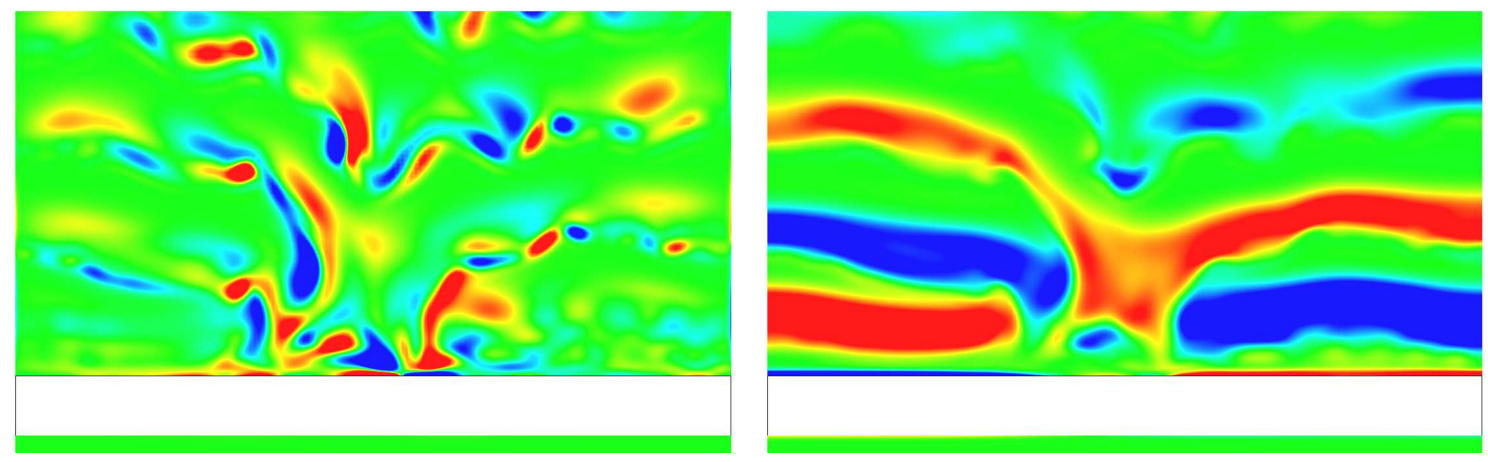

(c)

Figure 17: Iso-vorticity surfaces along $y=0$ of cross-stream $\left(\left|\omega_{y}\right|\right.$, left $)$ and spanwise $\left(\left|\omega_{z}\right|=1\right.$, right) vorticity for $\operatorname{Re}=200\left(L_{z}=12, x \in[-0.7,6.6], z \in[-6,6]\right.$; flow from below). (a): $t=463\left(\left|\omega_{y}\right|=0.4\right) ;(\mathrm{b})$ : $t=974\left(\mathrm{HF},\left|\omega_{y}\right|=1.0\right) ;(\mathrm{c}): t=928\left(\mathrm{LF},\left|\omega_{y}\right|=1.0\right)$.

\section{Concluding Remarks}

Flow past a stationary circular cylinder was simulated for $\mathrm{Re}=50-200$, using a finite volume code employing an implicit fractional step method with second-order accuracy in 
both space and time. Several cases were carried out to study the numerical parameters that may affect local or global results. The most sensitive flow quantity regarding the domain size was proven to be base pressure, followed by r.m.s. lift. In contrast, the streamwise wake bubble length and the separation angle were not affected by the domain size. Using a square domain, the necessary value for obtaining independent results in our study was $H=120$ at Re $=100$. The comparisons among different grid density both in the circumferential and radial directions demonstrated that r.m.s. drag was the most sensitive quantity, followed by r.m.s. lift and wake bubble length, which were especially sensitive to the grid density in the radial direction. As expected, there should be a high enough grid density along the cylinder surface, within the separated shear layer regions and inside the very near wake. It should be noted that r.m.s. force coefficients were rather sensitive to the time step size, especially r.m.s. drag coefficient, for which an increase of $16 \%$ can be observed when the time step size was increased from 0.005 to 0.04 . The wake bubble length was also somewhat sensitive to the time step size, as well as the base pressure. In this study, a time step of 0.01 was sufficiently short to capture the local and global features for 2-D shedding flow.

With respect to the effect of Reynolds number, global time-averaged results were analyzed in detail and compared with previous experimental and reliable numerical results. In addition, corresponding values distributed along the wake center line and cylinder surface were presented and discussed for $\operatorname{Re}=50,100,150,200$. Regarding the huge drop of the wake bubble length, by about $65 \%$ within $R e=50-200$, the stretching effect of viscous shear stresses and increasing time-averaged normal stresses due to shedding-related velocity fluctuations were believed to be the main contributing factors with respect to pushing the bubble towards the cylinder. A comparison of drag coefficients revealed that the frictional part of the (total) drag coefficient drops with Re roughly as $C / \sqrt{\operatorname{Re}}$, where $C=3.21$ at $\operatorname{Re}=50$, and $C=3.51$ at $\operatorname{Re}=200$. The ratio between frictional r.m.s. lift and total r.m.s. lift was found to decrease with increasing Reynolds number approximately as $1.3 / \sqrt{\operatorname{Re}}$. Within $\operatorname{Re}=50-200$ and still 2-D flow, an almost constant phase shift, $\phi \simeq 30^{\circ}$, was found from the force signals due to friction and pressure, presumably related to the maximum wall friction occurring upstream of the shoulder area, where the lift due to pressure has its main 
origin.

As to the time-averaged results' distribution along the wake centerline, the locations of corresponding peak values deviate from the wake closure points more at lower Reynolds numbers, while coincidence can be observed for $\mathrm{Re}=200$. Good agreement and similar trends were achieved in flow quantities distributed around the cylinder surface, in comparison with previous numerical and experimental results. A maximum (time-averaged) wall vorticity appeared at around $\theta=50^{\circ}$, whereas a maximum absolute pressure gradient occurred at around $\theta=40^{\circ}$ for all Reynolds number cases. As for the angular positions of maximum (time-averaged) wall pressure gradient and maximum r.m.s. wall pressure, both being used previously as measures for the (time-averaged) location of flow separation at higher Re with turbulent shedding conditions, it can be noted that, with laminar shedding, both these positions were significantly upstream of the actual flow separation, although with a decreasing difference with increasing Re.

Regarding the streamwise mean velocity deficit in the intermediate wake region, a local maximum value can be observed for $\operatorname{Re}>50$ between the range of $4<x<32$. That is to say, for all $\operatorname{Re} \geq 60$, there was a non-monotonous recovery of the time-averaged streamwise velocity along the wake center line $(y=0, x>0.5)$; for $\operatorname{Re} \geq 100$, the final monotonous recovery was initiated at $x=32 \pm 2$. Comparisons with related experimental and 2-D numerical results for the time-averaged streamwise velocity were made for $\operatorname{Re}=150$, showing disagreement in the region of $x<80$. For instance, the present 2-D results exhibited a local maximum wake velocity deficit of about 0.55 at $x \simeq 34$; previous related results indicate a local maximum at a velocity deficit of $0.38 \pm 0.02$ occurring at $x=44-50$. In addition, in the present results but not seen elsewhere, there was a distinct plateau for $\operatorname{Re}=150$ from $x=12$ to $x=20$ at a velocity deficit of about 0.37 . This distinct plateau behavior was observed for all Re $>120$ and occurred within $x=9-20$ at around the same velocity deficit $(0.37-0.38)$. The study of the primary vortex street evolution revealed that the appearance of the first wake velocity deficit observed for Re $>50$ was due to the von Kármán vortices quickly moving away from the wake centerline. As for the plateau-like behavior occurring for Re $>100$, distortion and turning of the von Kármán vortices were thought to be the main 
reason. In particular, a merging trend of the von Kármán vortices appearing for $\operatorname{Re}=200$ beyond $x=25$ was believed to contribute to the formation of the second plateau behavior.

Finally, for the 3-D simulations at $\operatorname{Re}=200\left(L_{z}=10\right.$ and $\left.L_{z}=12\right)$ and comparing with 2-D simulation results, significant changes in most global quantities were observed. Good agreement with previous experiments and reliable numerical results was obtained after a rather long simulation time until the presumably ultimate and much more chaotic state was reached, referred to as mode $\mathrm{A}^{*}$ in Williamson (1992). In the high force regions, the near wake flow exhibited mode A instability structures, but not as regular as in the initial flowdeveloping region. Present 3-D results as well as those of previous studies at this particular $\operatorname{Re}=200$ suggest that a spanwise length of at least $L_{z}=12$ is needed to reach a final flow state in good agreement with experiments.

\section{Acknowledgements}

This work was mainly carried out during the first author's stay at Chalmers University of Technology. The first author was financially supported by the China Scholarship Council and National Natural Science Foundation of China (No. 51139007), both of which are appreciated very much. The financial support of SNIC (Swedish National Infrastructure for Computing) for computer time at C3SE (Chalmers Center for Computational Science and Engineering) is also gratefully acknowledged. The first author is also grateful to Prof. Lars-Erik Eriksson, Haukur Hafsteinsson and Lars Ellbrant for their support and help in using G3DMESH. Many thanks to Jelena Andric, Olof Penttinen and Atilla Altintas for their kind assistance.

\section{References}

Balachandar, S., Mittal, R., Najjar, F. M., 1997. Properties of the mean recirculation region in the wakes of two-dimensional bluff bodies. Journal of Fluid Mechanics 351, 167-199.

Baranyi, L., Lewis, R. I., 2006. Comparison of a grid-based CFD method and vortex dynamics predictions of low Reynolds number cylinder flows. The Aeronautical Journal 110 (1103), 63-71.

Baranyi, L., Shirakashi, M., 1999. Numerical solution for laminar unsteady flow about fixed and oscillating cylinders. Journal of Computer Assisted Mechanics and Engineering Sciences 6, 263-277.

Barkley, D., Henderson, R. D., 1996. Three-dimensional Floquet stability analysis of the wake of a circular cylinder. Journal of Fluid Mechanics 322, 215-241. 
Behara, S., Mittal, S., 2009. Parallel finite element computation of incompressible flows. Parallel Computing 35, 195-212.

Behr, M., Hastreiter, D., Mittal, S., Tezduyar, T. E., 1995. Incompressible flow past a circular cylinder: Dependence of the computed flow field on the location of the lateral boundaries. Computer Methods in Applied Mechanics and Engineering 123, 309-316.

Berthelsen, P. A., Faltinsen, O. M., 2008. A local directional ghost cell approach for incompressible viscous flow problems with irregular boundaries. Journal of Computational Physics 227 (9), 4354-4397.

Braza, M., Chassaing, P., Minh, H. H., 1986. Numerical study and physical analysis of the pressure and velocity fields in the near wake of a circular cylinder. Journal of Fluid Mechanics 165, 79-130.

Braza, M., Faghani, D., Persillon, H., 2001. Successive stages and the role of natural vortex dislocations in three-dimensional wake transition. Journal of Fluid Mechanics 439, 1-41.

Cao, S., Ozono, S., Tamura, Y., Ge, Y., Kikugawa, H., 2010. Numerical simulation of Reynolds number effects on velocity shear flow around a circular cylinder. Journal of Fluids and Structures 26, 685-702.

Carmo, B. S., Meneghini, J. R., 2006. Numerical investigation of the flow around two circular cylinders in tandem. Journal of Fluids and Structures 22, 979-988.

Cimbala, J. M., 1984. Large structure in the far wakes of two-dimensional bluff bodies. Ph.D. thesis, California Institute of Technology, California.

Davidson, L., Farhanieh, B., 1995. CALC-BFC: A finite-volume code employing collocated variable arrangement and cartesian velocity components for computation of fluid flow and heat transfer in complex three-dimensional geometries. Rept. 95/11, Thermo and Fluid Dynamics, Chalmers University of Technology, Gothenburg, Sweden.

Davidson, L., Peng, S. H., 2003. Hybrid LES-RANS modelling: a one-equation SGS model combined with a $k-\omega$ model for predicting recirculating flows. International Journal for Numerical Methods in Fluids 43, $1003-1018$.

Eames, I., Jonsson, C., Johnson, P. B., 2011. The growth of a cylinder wake in turbulent flow. Journal of Turbulence 12 (39), 1-16.

Eisenlohr, H., 1990. Ein kurzer oder ein langer Zylinder: Worin liegt der Unterschied für die Kármánsche Wirbelstrasse? Mitteilungen Nr. 98, Max-Planck-Institut für Strömungsforschung, Göttingen.

Emvin, P., 1997. The full multigrid method applied to turbulent flow in ventilated enclosures using structured and unstructured grids. Ph.D. thesis, Chalmers University of Technology, Sweden.

Eriksson, L. E., 1985. Practical three-dimensional mesh generation using transfinite interpolation. SIAM Journal on Scientific Computing 6 (3), 712-741.

Fey, U., König, M., Eckelmann, H., 1998. A new Strouhal-Reynolds-number relationship for the circular cylinder in the range $47<\operatorname{Re}<2 \times 10^{5}$. Physics of Fluids 10 (7), 1547-1549. 
Franke, R., Rodi, W., Schönung, B., 1990. Numerical calculation of laminar vortex-shedding flow past cylinders. Journal of Wind Engineering and Industrial Aerodynamics 35, 237-257.

Gioria, R. S., Meneghini, J. R., Aranha, J. A. P., Barbeiro, I. C., Carmo, B. S., 2011. Effect of the domain spanwise periodic length on the flow around a circular cylinder. Journal of Fluids and Structures 27, $792-797$.

Henderson, R. D., 1995. Details of the drag curve near the onset of vortex shedding. Physics of Fluids 7 (9), $2102-2104$.

Henderson, R. D., 1997. Nonlinear dynamics and pattern formation in turbulent wake transition. Journal of Fluid Mechanics 352, 65-112.

Homann, F., 1936a. Der Einfluss grosser Zähigkeit bei der Strömung um den Zylinder und um die Kugel. Zeitschrift für angewandte Mathematik und Mechanik 16 (3), 153-164.

Homann, F., 1936b. Einfluss grosser Zähigkeit bei Strömung um Zylinder. Forschung auf dem Gebiete Ingenieurwesens 7 (1), 1-10.

Inoue, O., Yamazaki, T., 1999. Secondary vortex streets in two-dimensional cylinder wakes. Fluid Dynamics Research 25, 1-18.

Kang, S., 2006. Uniform-shear flow over a circular cylinder at low Reynolds numbers. Journal of Fluids and Structures 22, 541-555.

Karasudani, T., Funakoshi, M., 1994. Evolution of a vortex street in the far wake of a cylinder. Fluid Dynamics Research 14, 331-352.

Kravchenko, A. G., Moin, P., 1998. B-spline methods and zonal grids for numerical simulations of turbulent flows. Report No. TF-73, Flow Physics and Computational Division, Department of Mechanical Engineering, Stanford University, Stanford, California 94305.

Kumar, B., Mittal, S., 2006. Effect of blockage on critical parameters for flow past a circular cylinder. International Journal for Numerical Methods in Fluids 50, 987-1001.

Labbé, D. F. L., Wilson, P. A., 2007. A numerical investigation of the effects of the spanwise length on the 3-D wake of a circular cylinder. Journal of Fluids and Structures 23, 1168-1188.

Leontini, J. S., Thompson, M. C., Hourigan, K., 2010. A numerical study of global frequency selection in the time-mean wake of a circular cylinder. Journal of Fluid Mechanics 645, 435-446.

Li, Y., Zhang, R., Shock, R., Chen, H., 2009. Prediction of vortex shedding from a circular cylinder using a volumetric Lattice-Boltzmann boundary approach. The European Physical Journal Special Topics 171, 91-97.

Lomtev, I., Quillen, C. B., Karniadakis, G. E., 1998. Spectral/hp methods for viscous compressible flows on unstructured 2D meshes. Journal of Computational Physics 144, 325-357.

Miller, G. D., Williamson, C. H. K., 1994. Control of three-dimensional phase dynamics in a cylinder wake. 
Experiments in Fluids 18, 26-35.

Mittal, S., 2001. Computation of three-dimensional flows past circular cylinder of low aspect ratio. Physics of Fluids 13, 177-191.

Mittal, S., 2005. Excitation of shear layer instability in flow past a cylinder at low Reynolds number. International Journal for Numerical Methods in Fluids 49 (10), 1147-1167.

Norberg, C., 1987. Effects of Reynolds number and a low-intensity freestream turbulence on the flow around a circular cylinder. Publication 87/2, Dept. Applied Thermodynamics and Fluid Mechanics, Chalmers University of Technology, Gothenburg, Sweden.

Norberg, C., 1994. An experimental investigation of the flow around a circular cylinder: Influence of aspect ratio. Journal of Fluid Mechanics 258, 287-316.

Norberg, C., 1998. LDV-measurements in the near wake of a circular cylinder. In: Bearman, P. W., Williamson, C. H. K. (Eds.), Advances in the Understanding of Bluff Body Wakes and Vortex-Induced Vibration, Washington, D.C. ASME, New York, pp. 1-12.

Norberg, C., 2001. Flow around a circular cylinder: Aspects of fluctuating lift. Journal of Fluids and Structures 15, 459-469.

Norberg, C., 2002. Pressure distributions around a circular cylinder in cross-flow. In: Hourigan, K., Leweke, T., Thompson, M. C., Williamson, C. H. K. (Eds.), Symposium on Bluff Body Wakes and Vortex-Induced Vibrations (BBVIV3), 17-20 Dec. 2002, Port Arthur, Queensland, Australia, 2002. Monash University, Melbourne, Australia, pp. 1-4.

Norberg, C., 2003. Fluctuating lift on a circular cylinder: Review and new measurements. Journal of Fluids and Structures 17, 57-96.

Park, J., Kwon, K., Choi, H., 1998. Numerical solutions of flow past a circular cylinder at Reynolds numbers up to 160. KSME International Journal 12 (6), 1200-1205.

Persillon, H., Braza, M., 1998. Physical analysis of the transition to turbulence in the wake of a circular cylinder by three-dimensional Navier-Stokes simulation. Journal of Fluid Mechanics 365, 23-88.

Ponta, F. L., 2010. Vortex decay in the Kármán eddy street. Physics of Fluids 22 (093601), 1-11.

Posdziech, O., Grundmann, R., 2001. Numerical simulation of the flow around an infinitely long circular cylinder in the transition regime. Theoretical and Computational Fluid Dynamics 15, 121-141.

Posdziech, O., Grundmann, R., 2007. A systematic approach to the numerical calculation of fundamental quantities of the two-dimensional flow over a circular cylinder. Journal of Fluids and Structures 23 (3), 479-499.

Rajani, B. N., Kandasamy, A., Majumdar, S., 2009. Numerical simulation of laminar flow past a circular cylinder. Applied Mathematical Modelling 33, 1228-1247.

Relf, E. F., 1914. Discussion of the results of measurements of the resistance of wires, with some additional 
tests on the resistance of wires of small diameter. Reports and Memoranda No. 102, Advisory Committee for Aeronautics (ACA).

Roshko, A., 1954. On the development of turbulent of turbulent wake from vortex streets. NACA Report 1191, National Advisory Committee for Aeronautics, Washington, D.C.

Schlichting, H., 1979. Boundary-Layer Theory, 7th Edition. McGraw-Hill, New York.

Sears, W. R., Telionis, D. P., 1975. Boundary-layer separation in unsteady flow. SIAM Journal on Applied Mathematics 28 (1), 215-235.

Sharman, B., Lien, F. S., Davidson, L., Norberg, C., 2005. Numerical predictions of low Reynolds number flows over two tandem circular cylinders. International Journal for Numerical Methods in Fluids 47, 423-447.

Shi, J. M., Gerlach, D., Breuer, M., Biswas, G., Durst, F., 2004. Heating effect on steady and unsteady horizontal laminar flow past a circular cylinder. Physics of Fluids 16 (12), 4331-4345.

Sohankar, A., Norberg, C., Davidson, L., 1998. Low-Reynolds-number flow around a square cylinder at incidence: study of blockage, onset of vortex shedding and outlet boundary condition. International Journal for Numerical Methods in Fluids 26, 39-56.

Sohankar, A., Norberg, C., Davidson, L., 1999. Simulation of three-dimensional flow around a square cylinder at moderate Reynolds number. Physics of Fluids 11 (2), 288-306.

Stålberg, E., Brüger, A., Lötstedt, P., Johansson, A. V., Henningsson, D. S., 2006. High order accurate solution of flow past a circular cylinder. Journal of Scientific Computing 27 (1-3), 431-441.

Thom, A., 1928. An investigation of fluid flow in two dimensions. Reports and Memoranda 1194, Aeronautical Research Committee, London, U.K.

Thom, A., 1933. The flow past circular cylinders at low speeds. Proceedings of the Royal Society (London), Series A 141, 651-669.

Thompson, M. C., Leweke, T., Williamson, C. H. K., 2001. The physical mechanism of transition in bluff body wakes. Journal of Fluids and Structures 15, 607-616.

Tritton, D. J., 1959. Experiments on the flow past a circular cylinder at low Reynolds numbers. Journal of Fluid Mechanics 6 (4), 547-567.

Vorobieff, P., Georgiev, D., Ingber, M. S., 2002. Onset of the second wake: Dependece on the Reynolds number. Physics of Fluids 14 (7), L53-56.

White, F. M., 1991. Viscous Fluid Flow, 2nd Edition. McGraw-Hill, New York.

Wieselsberger, C., 1921. Neuere Festellungen über die Gesetze des Flüssigkeits- und Luftwiderstandes. Physikalische Zeitschrift 22 (11), 321-328.

Williamson, C. H. K., 1988. The existence of two stages in the transition to three-dimensionality of a cylinder wake. Physics of Fluids 31 (11), 3165-3168. 
Williamson, C. H. K., 1989. Oblique and parallel modes of vortex shedding in the wake of a circular cylinder at low Reynolds numbers. Journal of Fluid Mechanics 206, 579-627.

Williamson, C. H. K., 1992. The natural and forced formation of spot-like 'vortex dislocations' in the transition of a wake. Journal of Fluid Mechanics 243, 393-441.

Williamson, C. H. K., 1995. Vortex dynamics in the wake of a cylinder. In: Green, S. I. (Ed.), Fluid Vortices. Kluwer Academic Publishers, Amsterdam, Holland, pp. 155-234.

Williamson, C. H. K., 1996a. Mode A secondary instability in wake transition. Physics of Fluids 8 (6), 1680-1682.

Williamson, C. H. K., 1996b. Vortex dynamics in the cylinder wake. Annual Review of Fluid Mechanics 28, $477-539$.

Williamson, C. H. K., Brown, G. L., 1998. A series in $1 / \sqrt{\text { Re }}$ to represent the Strouhal-Reynolds number relationship of the cylinder wake. Journal of Fluids and Structures 12, 1073-1085.

Williamson, C. H. K., Prasad, A., 1993. A new mechanism for oblique wave resonance in the 'natural' far wake. Journal of Fluid Mechanics 256, 269-313.

Williamson, C. H. K., Roshko, A., 1990. Measurements of base pressure in the wake of a cylinder at low Reynolds numbers. Zeitschrift für Flugwissenschaften und Weltraumforschung 14, 38-46.

Wu, M. H., Wen, C. Y., Yen, R. H., Weng, M. C., Wang, A. B., 2004. Experimental and numerical study of the separation angle for flow around a circular cylinder at low Reynolds number. Journal of Fluid Mechanics 515, 233-260.

Yoshida, T., Watanabe, T., Nakamura, I., 1993. Numerical analysis of open boundary conditions for incompressible viscous flow past a square cylinder. Transactions of the Japan Society of Mechanical Engineers, Series B 59 (565), 2799-2806. 